\title{
IMPLICACIÓN DE LA COCAÍNA EN LA PATOLOGÍA VASCULAR CEREBRAL
}

DEPARTAMENTO DE MEDICINA

UNIVERSITAD AUTÓNOMA DE BARCELONA

AUTORA: MARÍA E. CARCELÉN GADEA

DIRECTOR DEL TRABAJO: JOSÉ ALVAREZ SABIN

TRABAJO DE INVESTIGACIÓN

AÑO 2010

CONVOCATORIA JUNIO 


\section{ÍNDICE}

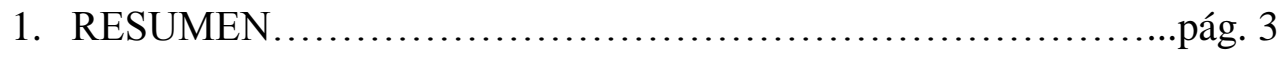

2. REVISIÓN Y ACTUALIZACIÓN DEL TEMA.......................4

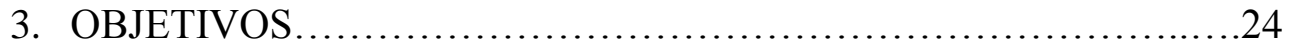

4. MATERIAL Y MÉTODOS .......................................25

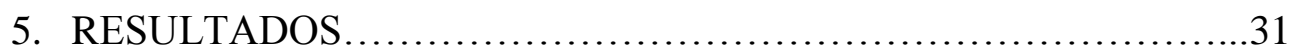

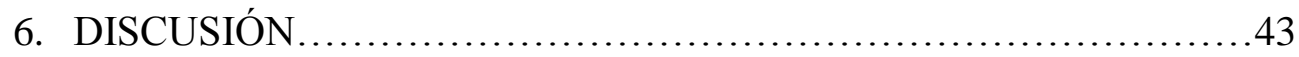

7. CONCLUSIONES............................................... 46

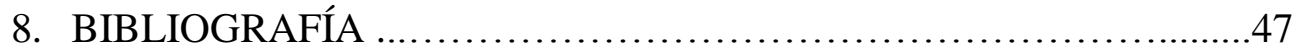

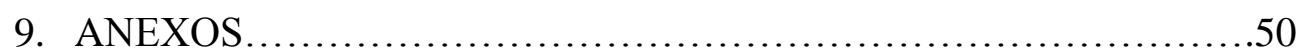




\section{RESUMEN}

\section{OBJETIVO}

Estudio descriptivo, cuyo objetivo principal es describir la implicación de la cocaína en la patología vascular cerebral en pacientes jóvenes.

\section{MATERIAL Y MÉTODOS}

Revisión retrospectiva de pacientes menores de 50 años ingresados en el servicio de Neurología con patología cerebrovascular aguda desde Enero 2006 hasta Diciembre 2009. Se comparan 18 pacientes con niveles de cocaína positivos al ingreso y 79 pacientes con niveles negativos. De todos ellos, se recogen distintas variables que definen el perfil de riesgo vascular, características del ictus y morbimortalidad asociada a los mismos; finalmente se realiza un análisis estadístico de ambas poblaciones.

\section{RESULTADOS}

Se obtiene un predominio de varones, más evidente en el grupo control (55.6\% vs 64.6\%), no existen diferencias en estancia hospitalaria y en el grupo consumidor de cocaína destaca: edad media menor $(35.22 \pm 8.99 \mathrm{~S}$ vs $41.53 \pm 7.73 \mathrm{~S})$, consumo de otros tóxicos (tabaco, alcohol y cannabis) y frecuencia de trastorno psiquiátrico mayor (16.7\% vs $3.8 \%$ ), factores de riesgo cardiovascular (HTA, DM, DL) menor al ingreso (16.7\% vs $36.7 \%)$ y al alta (38.9\% vs $60.8 \%)$, hipercekaemia mayor (27.8\% vs $5.1 \%)$, menos hallazgos patológicos en pruebas dirigidas al estudio etiológico, más complicaciones neurológicas (33.3\% vs $15.2 \%$ ) y mortalidad (11.1\% vs $3.8 \%$ ) sin diferencias en morbilidad (Rankin y NIHSS). Diagnósticos: predomino isquemia y territorio anterior en ambos, AIT (11.1\% vs $12.7 \%)$, HIC (22.2\% vs $13.9 \%)$, HSA ( $16.7 \%$ vs $11.4 \%$ ), causa inhabitual (22.2\% vs $20.3 \%)$, indeterminado (27.8\% vs $20.3 \%)$, ningún caso de aterotrombótico, cardioembólico ni lacunar en grupo de cocaína.

\section{CONCLUSIONES}

La cocaína es un factor de riesgo evidente a tener en cuenta en adultos jóvenes, responsable de eventos neurológicos a edades más tempranas de lo esperado según la evolución natural con los habituales factores de riesgo vascular. Es necesaria la concienciación de los médicos y estudios prospectivos. 


\section{REVISIÓN DEL TEMA}

\section{INTRODUCCIÓN}

La cocaína se obtiene de las hojas del arbusto Erytroxylon coca, planta originaria de los Andes y que se cultiva en América del Sur, en Indonesia y en algún estado africano. Contiene hasta $14-17$ alcaloides distintos de los que el más conocido y estudiado es la cocaína [1].

Se presenta comúnmente en polvo, en forma de clorhidrato de cocaína. Penetra directamente en el sistema nervioso central a través del torrente sanguíneo produciendo una sensación agradable, anestésica y estimulante [1].

El consumo de cocaína en España se ha ido incrementando progresivamente en los últimos años, presentando una de las tasas mayores de consumo en la Unión Europea [9]. A partir de los años 90 el consumo de cocaína se extiende a todas las clases sociales incluidas las más bajas. El aumento del consumo está proporcionalmente ligado al aumento de ocio nocturno, un cambio en la escala de valores basada en el éxito social rápido y en la instauración de una moda de consumo recreativo de drogas [1].

Cerca de un $90 \%$ de los adictos a la cocaína también consumen alguna otra droga, sobre todo depresores como el alcohol, la marihuana y la heroína, aunque el consumo único de cocaína se ha visto incrementado en los últimos años [1, 2].

$\mathrm{El}$ aumento en el consumo ha llevado a un aumento en la incidencia de urgencias médicas y psiquiátricas secundarias al consumo y de la patología orgánica derivada del mismo $[2,12]$.

\section{EPIDEMIOLOGÍA}

Según la última encuesta domiciliaria sobre abuso de drogas en España (EDADES), realizada por el Observatorio español de drogas en 2007 [13], la cocaína es la segunda droga psicoactiva ilegal en cuanto a prevalencia de consumo en España.

En 2007 el 8\% de la población de 15-64 años había probado alguna vez cocaína en polvo, el 3,0\% lo había hecho durante el último año y un 1,6\% el último mes. La prevalencia de consumo durante los últimos 12 meses fue más elevada en hombres $(4,7 \%)$ que en mujeres $(1,6 \%)$ y en el grupo de $15-34$ años $(5,3 \%)$ que en el de 35-64 $(1,3 \%)$. En cuanto a la evolución temporal, la tendencia de consumo de cocaína en polvo parece estabilizada, de hecho la prevalencia aumentó entre 1995 (1,8\%) y 2005 (3\%), y en 2007 continúa en el 3\%. 
Desde 1999 y hasta 2005 se había producido un discreto incremento en la prevalencia de consumo de cocaína base alguna vez en la vida, sin embargo en 2007 se ha producido un aumento muy acusado, lo que nos informa de la mayor experimentación de esta forma de consumo. La edad media de primer consumo de esta sustancia se situó en 20,9 años para la cocaína en polvo en 2007. Con respecto a 1999 (21,8 años) se observa un adelanto en la edad media de inicio.

La continuidad en el consumo de cocaína no es elevada. En 2007 poco más de un tercio de los que habían probado esta droga alguna vez en la vida la habían consumido en los últimos 12 meses y aproximadamente un quinto la habían consumido en los últimos 30 días. Como se puede apreciar observando las curvas de prevalencia en los distintos períodos, la continuidad en el consumo ha disminuido en los últimos años.

El informe 2009 del Observatorio europeo de las drogas y las toxicomanías [12], señala que durante el último decenio se ha observado un aumento general del consumo y de incautación de cocaína en la Unión Europea, principalmente en Europa occidental (Dinamarca, España, Italia, Irlanda y el Reino Unido). Al comparar internacionalmente, la estimación de la prevalencia del consumo de cocaína durante el último año es más baja en los adultos jóvenes en la Unión Europea que dentro del mismo colectivo en Australia y los Estados Unidos. No obstante, dos países, España y el Reino Unido (Inglaterra y Gales) notifican unas cifras más elevadas que Australia, y sólo España indica una estimación más elevada que la de los Estados Unidos.

Los datos disponibles también indican una diversidad considerable entre consumidores de cocaína, tanto en términos de pautas como en términos socio demográficos. Debido a esta diversidad, evaluar la prevalencia del consumo de la droga, así como sus consecuencias sociales y para la salud resulta bastante complejo. La prevalencia de consumo a lo largo de la vida es de unos 13 millones de europeos (el 3,9 $\%$ de los europeos adultos) [12].

La red DA WN (American Drug Abuse Warning Network) estimó que la cocaína, por sí sola o en combinación con otras drogas, estaba implicada en seis de cada diez urgencias relacionadas con las drogas en 2006. En España, el sistema nacional de notificación de urgencias hospitalarias relativas al consumo no médico de sustancias psicotrópicas llegó a la conclusión de que, en 2006, la cocaína era la sustancia más frecuentemente consumida (en el $59 \%$ de los casos) [12]. Se ha producido un incremento de más de un $20 \%$ desde 1996 [1]. El hecho de que esté incrementando paulatinamente el número de episodios de urgencia hospitalaria y de muertes en los que 
está involucrada la cocaína, sirve para subrayar, por un lado el incremento de uso-abuso de cocaína y por otro, la potencial toxicidad de esta sustancia [2].

\section{FARMACOLOGÍA, FISIOPATOLOGÍA Y MECANISMOS DE ACTUACIÓN:}

Las formas de abuso de cocaína son de gran interés, ya que condicionan la farmacocinética, la actividad farmacológica, la toxicidad y el grado de adicción de la droga. Fundamentalmente se distinguen las siguientes formas de abuso: Hojas de coca, pasta de coca, clorhidrato de cocaína y cocaína base [3].

En nuestro medio, como se ha apuntado previamente, la forma más frecuente de consumo es el Clorhidrato de cocaína. Se trata de la sal de la cocaína formada con ácido clorhídrico. Se presenta en forma de micro cristales escamosos blancos, más o menos adulterada y se administra por vía intranasal (para esnifar) o se inyecta por vía venosa (no se puede fumar pues se destruye por el calor) [3].

La cocaína es muy lipofílica y atraviesa la barrera hematoencefálica de forma rápida y eficaz, encontrando niveles de cocaína en el cerebro 30 segundos tras ser esnifada o administrada por vía intravenosa ó en 5 segundos tras ser fumada [3, 8].

La vía intranasal es un modo muy común de usar la cocaína. Debido a la intensa vascularización de la mucosa de la nasofaringe la absorción es rápida así como sus efectos (locuacidad, sensación de energía) que duran entre 20 y 40 minutos. La biodisponibilidad nasal u oral es de un 30-40\%, aunque la variabilidad es mayor para la vía oral. Es la forma de consumo de cocaína con menor toxicidad aguda potencial, si exceptuamos la forma de consumo de los mascadores de hojas [3].

La vía endovenosa también es frecuente. A veces se combina con heroína (speed ball) para evitar los efectos de rebote desagradables producidos por la cocaína. Los efectos farmacológicos y psíquicos por cocaína endovenosa son inmediatos (30 segundos) y potentes pero de breve duración (10-20 minutos), con aparición posterior de un intenso crash (disforia, irritabilidad y alteraciones gastrointestinales) [3].

La benzoilecgonina es el metabolito que se detecta en orina y más utilizado para monitorizar los tratamientos. Puede ser detectada en orina 3-4 días después del último consumo (hasta diez en consumidores crónicos) y por supuesto dependerá de la cantidad de cocaína consumida y del valor de corte que se establezca o de la sensibilidad de la prueba. La vía de administración también influye en la cantidad de benzoilecgonina que se detecta en plasma y que se eliminará a través de la orina $[3,8]$. 
Entre las acciones farmacológicas de la cocaína cabe destacar su acción como anestésico local que se produce al bloquear la transmisión nerviosa por alteración de la permeabilidad iónica de las membranas y su acción como estimulante del SNC, debida fundamentalmente al bloqueo de la recaptación presináptica de catecolaminas y serotonina por unión al transportador de recaptación de aminas (DAT) dando como resultado un exceso de neurotransmisor en la hendidura sináptica $[1,3,4,14]$.

Según esto, la cocaína se comporta como una amina simpaticomimética de acción indirecta, es decir, es capaz de remedar las acciones de las catecolaminas no actuando directamente sobre los receptores adrenérgicos o dopaminérgicos, sino aumentando la disponibilidad del neurotransmisor en la hendidura sináptica [3].

Se diferencian tres efectos fundamentalmente:

$\checkmark$ Agonista Dopaminérgico: Bloqueo de la recaptación e inducción de la síntesis de dopamina, aumentando la actividad dopaminérgica, particularmente en el área tegmental ventral (ATV) y en el núcleo accumbens, es decir, en las principales áreas del sistema dopaminérgico mesocorticolímbico. También incrementa la actividad glutamatérgica en el núcleo accumbens, el ATV, el estriado y la corteza prefrontal [4], [15]. Estas vías dopaminérgicas y el aumento de la biodisponibilidad de dopamina están relacionadas con los sistemas de recompensa en el cerebro, la sensibilización y el mecanismo de adicción [3], [4].

Según Fernández-Espejo [4], el incremento en la actividad de dopamina en el ATV tras psicoestimulantes, facilitada por despolarización neuronal, estimula receptores D1 presinápticos que ocasionan una mayor liberación local de glutamato, lo que a su vez estimula aún más las neuronas de dopamina del ATV, constituyendo estos hechos un ciclo fundamental en la inducción de la sensibilización a psicoestimulantes. Una mayor actividad glutamatérgica sobre el núcleo accumbens, podría facilitar cambios plásticos en forma de circuitos funcionales nuevos o engramas, cuya activación podría ser la base neuronal de la 'motivación' por la droga. Todos estos hechos neuroquímicos concuerdan con la teoría actual sobre la adicción que combina un aumento de respuestas condicionadas asociadas al uso de la droga, junto a una pérdida de control inhibitorio que daría lugar a la impulsividad del consumo. La transición desde el consumo controlado de droga hasta el hábito compulsivo depende de modo crítico de la pérdida del control ejecutivo de la corteza prefrontal sobre el estriado dorsal. 
Además de los efectos comentados, el cambio en los niveles de dopamina por el bloqueo de la recaptación de ésta, van a influir en la regulación del flujo sanguíneo cerebral, ya que la dopamina puede inducir vasoespasmo del músculo liso vascular cerebral con una probable acción en el control del flujo sanguíneo cerebral. El efecto vasoconstrictor provocado por la recaptación de otras aminas como la norepinefrina y la serotonina contribuirán a la reducción del flujo sanguíneo cerebral. Este efecto persistirá en el tiempo debido a la presencia de metabolitos activos [8, 16].

$\checkmark$ Agonista adrenérgico: los dos metabolitos principales de la cocaína, la benzoilecgonina (BEG) y la ecgoninametilester (EME) bloquean el sistema de transporte neuronal, impidiendo la recaptación. El exceso de noradrenalina va a permitir una estimulación mayor y más prolongada de los receptores adrenérgicos $\alpha$ y $\beta$ e hiperactividad del sistema nervioso autónomo simpático, responsable de la mayoría de los efectos farmacológicos y de las complicaciones agudas de la cocaína [3].

Por tanto, el consumo de cocaína produce:

- Vasoconstricción arterial por dos mecanismos, indirectamente, inhibiendo la recaptación de catecolaminas y serotonina, y directamente, actuando sobre el flujo de calcio y los canales de sodio [11, 17].

En la circulación cerebral el efecto de la cocaína es constrictor o dilatador dependiendo de los receptores postsinápticos de cada territorio arterial como se refleja en los estudios experimentales realizados en conejos [16], que mostraron una clara evidencia de alteración en la vasoregulación inducida por la cocaína, con vasoespasmo o vasodilatación. En el estudio angiográfico cerebral realizado a los conejos tras inyección iv de cocaína, se observó que el segmento P1 de la arteria cerebral posterior prácticamente está exento de inervación simpática, si bien, previamente había sido descritos [20] infartos talamomesencefálicos por oclusión de esta rama arterial relacionados con el consumo de cocaína, en los que la hiperactividad adrenérgica no explicaría su mecanismo de producción. Los receptores serotoninérgicos podrían estar implicados.

Además, en un ensayo clínico aleatorizado doble ciego [21], en el que se administró a una serie de pacientes cocaína intravenosa a diferentes dosis vs placebo, se concluyó que el efecto vasoconstrictor comprobado mediante angioresonancia también depende de la dosis empleada. 
En el trabajo publicado en 1999 por Blanco y Diez-Tejedor [11] sobre la cocaína y enfermedad cerebrovascular en adultos jóvenes, existía un predominio de lesiones en la circulación anterior sobre la posterior, que se podría explicar por esa diferencia de receptores en cada territorio arterial expuesto previamente.

Más recientemente en 2005, Johnson et al [34], han publicado un estudio doble ciego aleatorizado en seres humanos con trastorno por consumo de cocaína; dividen un total de 31 pacientes en 1 grupo que recibe cocaína a dosis bajas vs placebo y otro grupo que recibe cocaína a dosis altas vs placebo, y evalúan cuantitativamente el flujo sanguíneo cerebral mediante tomografía por emisión de positrones (PET). Concluyendo que la dosis alta de cocaína se asocia con una mayor hipoperfusión alcanzando significación estadística sólo para las regiones ricas dopamina y las regiones cerebrales medias. Además, a pesar de que la hipoperfusión ocurre en todo el cerebro, la región hemisférica izquierda rica en dopamina fue la más afectada.

- Hipertensión arterial, por su efecto inotrópico y cronotrópico positivo unido al efecto vasoconstrictor [3]. El aumento agudo de la tensión arterial tras la administración de cocaína contribuye a la lesión endotelial y podría estar relacionado con la presencia de infartos subcorticales dependientes de ramas lenticuloestriadas que son más sensibles a los efectos de la HTA [44]. En el caso de las hemorragias, la ruptura de la pared arterial se ha relacionado con la hipertensión sistémica entre otros factores. Por otra parte, la vasoconstricción puede ir seguida de reperfusión del cerebro, inicialmente isquémico, y producir una hemorragia secundaria al infarto [17].

- Taquicardia por estímulo sinusal, como consecuencia directa del estímulo simpático, tanto central como periférico. A dosis bajas puede existir bradicardia por depresión del nodo sinusal [3]. Además a nivel cardiaco se produce un aumento de la fuerza de contracción y de la frecuencia cardiaca por un estímulo de los receptores b1 fundamentalmente [3].

- Crisis convulsivas [3, 6, 8]. El mecanismo fisiopatológico no está aclarado completamente pero se relaciona con el bloqueo de la recaptación de catecolaminas [8]. Algunas hipótesis hacen referencia a la posible contribución del efecto la anestésico local de la cocaína [4].

- Otros efectos por estímulo simpático son la midriasis, temblor, sudoración, contracción de esfínteres y retraso digestivo [3, 4]. 
Agonista serotoninérgico: inhibe la recaptación de la serotonina y de su precursor el triptófano dentro de las neuronas serotoninérgicas. Esto lleva a la estimulación de los autoreceptores inhibitorios presinápticos aumentando la concentración de la 5-HT en la hendidura; llevando a una retroalimentación negativa que causa un agotamiento rápido de 5-HT en el cerebro. En general el efecto sobre la 5-HT es inhibitorio. Además de los efectos vasoconstrictores ya comentados, esta vía estaría relacionada con los efectos alucinatorios y psicomiméticos pudiendo explicar los cambios motores y estereotipias vistos en personas intoxicadas. Recientemente, se están realizando numerosos estudios que intentan hallar una diana terapéutica para la deshabituación de cocaína; uno de estos estudios realizado en ratas [27] plantea la hipótesis de que los receptores de serotonina pueden ser una nueva diana para el desarrollo de medicamentos para la dependencia de la cocaína.

Además de su acción sobre los neurotransmisores citados, la cocaína provoca otros efectos que se citan a continuación:

Aumento de la temperatura corporal: Además del aumento de la producción de calor por aumento de la actividad muscular y de la disminución de su pérdida por la vasoconstricción, la cocaína aumenta la temperatura corporal por pérdida del control dopaminérgico de receptores hipotalámicos reguladores de la temperatura, por agotamiento de los depósitos de dopamina, con hipertermia de rebote [3].

$\checkmark$ Aumento del catabolismo energético: paralelamente con la descarga adrenérgica que se produce inicialmente, también se produce una estimulación de la enzima adenilciclasa y por tanto un aumento del segundo mensajero AMPc a partir del adenosin trifosfato (ATP), esto genera una respuesta adrenérgica caracterizada por una aceleración general de las funciones fisiológicas.

$\checkmark$ Efecto trombogénico directo ya que aumenta el TXA2 e inhibe la AT III y la prostaciclina [11]. Heesch CM et al [47], realizaron un ensayo aleatorizado doble ciego cruzado para estudiar la activación plaquetaria a 14 voluntarios sanos, tras recibir cocaína ( $2 \mathrm{mg} / \mathrm{kg}$ por vía nasal) y tras placebo. Los autores concluyen que la exposición a cocaína causa activación plaquetaria, liberación de gránulos alfa, y formación de microagregados plaquetarios. Apoyan la opinión de que la cocaína, incluso a dosis relativamente bajas comúnmente autoadministradas por los consumidores ocasionales, pueden promover la trombosis y predisponen a eventos isquémicos. Los inhibidores de plaquetas deberían considerarse de inicio en pacientes con sospecha isquemia en relación de cocaína. 
Mecanismo inflamatorio [31] e incluso se ha realizado algún trabajo para intentar demostrar que la cocaína induce apoptosis de las células del músculo liso vascular cerebral ocasionando diferentes tipos de daños microvasculares cerebrales [36].

Existen varios artículos [31, 36, 43] que han sugerido la existencia de vasculopatía en consumidores de cocaína, aunque se trata de un tema todavía no aclarado, que no ha podido comprobarse en otros trabajos, como una revisión [38] de muestras seleccionadas de cerebros de 12 casos de hemorragia intracerebral o subaracnoidea relacionadas con la cocaína, donde las arteriolas intracraneales fueron normales o mostraron cambios inespecíficos. A partir de estas observaciones, los autores sugieren que se producen hemorragias intracraneales en ausencia de anomalías vasculares fácilmente detectable.

$\checkmark$ Efectos anorexizantes e inhibición del sueño al actuar directamente sobre el hipotálamo, el sistema reticular ascendente y en el cerebelo [1]

$\checkmark$ A nivel local, debido a su acción irritante, anestésica y vasoconstrictora provoca pequeñas lesiones en la mucosa nasal con aparición de escaras, úlceras y sangrado frecuente lo que añade un factor de riesgo en la transmisión de enfermedades infecciosas entre consumidores al compartir el billete con sangre contaminada.

\section{INTOXICACIÓN POR COCAÍNA Y TRATAMIENTO}

Tras conocer los mecanismos de acción de la cocaína puede resumirse que se trata de un alcaloide con alto potencial tóxico, actividad vasoconstrictora moderada y graves efectos psicotropos.

La reacción tóxica aguda por cocaína presenta una sintomatología que puede llegar a comprometer la vida del sujeto con una secuencia de acontecimientos muy similar a la intoxicación por otro tipo de psicoestimulantes. En el caso de la cocaína existen una serie de circunstancias que modifican las características e intensidad del cuadro tóxico (tipo de preparado, contaminantes que incluye el preparado, vía de consumo y ambiente de consumo) [5].

Diversos autores han descrito la sintomatología habitual de la intoxicación aguda por cocaína, existiendo una gran variabilidad entre individuos como se ha comentado previamente [3, 5]. Estos síntomas incluyen: elevación del estado de ánimo, sensación de mayor energía y lucidez, desinhibición eufórica, disminución del apetito, insomnio, mayor rendimiento en la realización de tareas, disminución de la sensación de fatiga, 
hiperactividad motora, verbal e ideativa, confusión, lenguaje incoherente, irritabilidad, enrojecimiento nasal, congestión ocular, ausencia de colaboración, negación de la causa, agresividad verbal, deterioro de la capacidad de juicio, grandiosidad, impulsividad, irresponsabilidad, generosidad atípica, hipersexualidad, conductas extrañas repetitivas de tipo compulsivo, inquietud psicomotora, movimientos estereotipados de boca y lengua, alucinaciones visuales y táctiles, alteraciones de la percepción, reacciones paranoides con posible desencadenamiento de psicopatologías latentes o compensadas.

Como consecuencia de este consumo pueden aparecer fiebre, hipertensión y complicaciones orgánicas principalmente cardiacas (arritmias e infarto agudo de miocardio) y neurológicas (convulsiones e ictus) pudiendo llegar a ocasionar la muerte del sujeto. $[5,6,8]$.

Pasado el efecto agudo aparece un periodo de cansancio, fatiga y disforia, más pronunciada cuanto más rápidos e intensos son los efectos producidos por la cocaína. Generalmente aparece el deseo de droga ("craving"). Estos efectos, como ya se ha comentado previamente, se deben fundamentalmente a la inhibición de la recaptación de dopamina. (3).

Dado que no existe un antídoto específico para la cocaína, el tratamiento es de tipo sintomático y de apoyo [3]. La intoxicación por cocaína en cualquiera de sus formas de administración se trataría con: 1/ diacepan para sedación del paciente, en caso de que sea necesario o si presenta convulsiones; no hay pruebas de que los medicamentos anticonvulsivos permitan prevenir las crisis epilépticas inducidas por la cocaína, por lo que hay que contraindicar su empleo para este fin, 2/ neurolépticos tipo butirofenonas (haloperidol) para los cuadros psicóticos, teniendo en cuenta que pueden disminuir el umbral epileptógeno; muchos individuos se recuperan espontáneamente en unas horas al cesar el consumo de cocaína, por lo que no suelen requerir tratamiento, 3/ antiarrítmicos (en general se desaconsejan los bloqueantes beta adrenérgicos ya que facilitarían el estímulo alfa por la cocaína lo que incrementaría los efectos vasoconstrictores e hipertensivos) y 4 / medidas generales y de reanimación que precise. $[3,4,7]$.

\section{COMPLICACIONES ORGÁNICAS POR COCAÍNA}

Las complicaciones neurológicas, junto a las cardiológicas, constituyen los principales eventos toxicológicos secundarios al consumo de cocaína $[8,9]$. 
No se puede olvidar el hecho del abuso concomitante de diferentes sustancias neurotóxicas, puesto que existirá una sumación de efectos tóxicos [8]. El consumo de alcohol por parte de los cocainómanos es muy frecuente, existen estudios que lo cifran hasta en un 99\%, ingestas simultaneas en un $77 \%$ e ingestas de alcohol en cada consumo de cocaína hasta en un 30\%. La razón del consumo simultáneo de ambas drogas no está del todo aclarada. La potenciación de la euforia por la ingesta de alcohol puede ser la base de esta asociación, aunque también se han señalado la disminución de efectos indeseables como los cuadros migrañosos inducidos por la cocaína [3]. Independientemente de las razones, la combinación supone un riesgo y un aumento de la morbi-mortalidad asociada a la cocaína, debido a la potenciación de efectos y metabolitos comunes (cocaetileno) [3, 8, 18, 19].

\section{Complicaciones neurológicas:}

$\checkmark$ Enfermedad cerebrovascular.-

El uso de cocaína se ha relacionado en secuencia temporal con la aparición de accidentes vasculares isquémicos (lacunares o territoriales) y hemorrágicos (hemorragias intraparenqimatosas y subaracnoideas), la mayoría de los cuales aparecen antes de transcurrida una hora del consumo de la droga aunque, también se han observado la aparición de ictus retardados en el tiempo [6, 9, 11], además pueden ocurrir tanto en consumidores ocasionales como habituales [11].

El mecanismo por el que ocurren no está totalmente aclarado y probablemente sea multifactorial: vasoespasmo-trombosis (efecto vasoconstrictor y dopaminérgico), HTA transitoria (lesión endotelial, disección arterial, ruptura de aneurismas), embolia de origen cardíaco (arritmias, miocardiopatía dilatada, cardiopatía isquémica y endocarditis infecciosa), embolización de material utilizado para la disolución de la droga en condiciones no estériles, efecto trombogénico directo (aumenta los niveles de tromboxano A2 e inhibe la prostaciclina y la antitrombinaIII, existiendo un aumento en el número de plaqueta, su activación y agregación), apoptosis y mecanismo inflamatorio (arteritis/vasculitis) son la principales hipótesis propuestas $[6,8,9,11,16,17,36,31$, 47].

Son numerosos los artículos [11, 25, 44, 45, 49] que relacionan el consumo de cocaína con la patología cerebrovascular, estando aceptado actualmente su papel como factor de riego a tener en cuenta sobre todo en adultos jóvenes [8]. La mayoría de ellos 
son estudios retrospectivos y con pocos pacientes por lo que siguen existiendo puntos sin aclarar y entre ellos encontraremos diferencias en cuanto a los resultados obtenidos.

La primera discordancia es si son más frecuentes las complicaciones hemorrágicas o las isquémicas ya que este dato varía según las series.

Según el estudio llevado a cabo por (Blanco et al.; 1999) [11] con datos del "Young Adults Stroke Registry" en pacientes menores de 45 años en los Estados Unidos, en el periodo comprendido entre 1989 hasta 1998 se identificó la cocaína como responsable del $7.60 \%$ de los ictus en adultos jóvenes. La edad media de los pacientes era de 28.3 años. De los 13 casos estudiados, 8 presentaron manifestaciones isquémicas ( 5 infartos, 2 TIAs y 1 encefalopatía con múltiples lesiones isquémicas), 4 presentaron hemorragias intraparenquimatosas y 1 hemorragia subaracnoidea. En ningún caso el estudio angiográfico pudo demostrar aneurismas o malformaciones arterio-venosas. La principal vía de administración de cocaína para estos pacientes fue la intranasal y el tiempo que los pacientes llevaban consumiendo cocaína hasta el accidente vascular varió de pocas horas a varios años. El $61 \%$ de los pacientes tenían historia de consumo de otras drogas.

Otra revisión retrospectiva [49] analizó 42 pacientes con abuso de la cocaína e ictus. En este caso 15 pacientes presentaron hemorragia y el resto (27) ictus isquémico. La edad media fue de 38 años para el accidente cerebrovascular (+ / - 8,5 SD); el número de hombres y mujeres fue (20:7), similar a lo observado en la hemorragia subaracnoidea (HSA), tras la ruptura del aneurisma. 9 tenían hemorragias intracerebrales, 6 tenían HSA con hemorragia intracerebral (ICH) / infarto. 4 se diagnosticaron de ataque isquémico transitorio, en 2 se halló oclusión carotídea, y en 1 flujo lento en el sistema vertebrobasilar. Quince eran conocidos hipertensos. La cocaína fue la sustancia principal en todos los pacientes; 7 utilizaban otras sustancias como la marihuana y la heroína. 3 pacientes tenían el VIH, 3 la hepatitis, 2 la sífilis, y 1 tuberculosis. 15 tenían análisis de orina positivo para metabolitos de cocaína, 2 tuvieron análisis después. 9 pacientes con HSA o HIC presentaron mala situación neurológica al ingreso y murieron. La intoxicación por cocaína se correlacionó con accidentes mortales cerebrovascular $(\mathrm{ACV})(\mathrm{p}<0,001)$ y puntuación baja en E Glasgow (GOS) ( $<<0,001)$. De estos datos, se concluye que el consumo de cocaína se correlacionó con ictus fatal y peor pronóstico.

Acaba de publicarse un estudio [25], que revisa un total de 5142 pacientes ingresados entre Julio 1998 y Julio 2008, e identifica consumo de cocaína en 96 pacientes con el objetivo de definir la fisiopatología y las manifestaciones de la 
enfermedad neurovascular relacionada con la cocaína. De los 96 casos, 45 (47\%) ictus isquémico o ataque isquémico transitorio (AIT), 26 (27\%) hemorragia intracerebral (HIC) y $25(26 \%)$ hemorragia subaracnoidea. En total, $61(63.5 \%)$ consumidores activos y $35(36.5 \%)$ antecedentes de consumo. Los usuarios activos más propensos a tener HIC en comparación con los usuarios anteriores $(37,7 \%$ v 8,6\%) y menos probabilidad de ictus isquémico o AIT (36,1\% v 65,7\%). La causa más común de ictus isquémico o AIT fue la aterosclerosis de las grandes arterias en 20 (44\%) pacientes. De los 25 casos la hemorragia subaracnoidea, 22 (88\%) fueron por aneurisma. Tras este análisis los autores concluyen que el ictus isquémico es una presentación común neurovascular en pacientes con una historia pasada de consumo de cocaína, a menudo como resultado de la enfermedad aterosclerótica; ni la vasculitis ni el vasoespasmo son una causa común de ictus en esta cohorte. La HIC es más común en los usuarios activos, tal vez debido a los picos agudos de la presión arterial. Son necesarios otros ensayos prospectivos para confirmar estos resultados.

Como ya se ha comentado, la cocaína es una de las causas de la hemorragia intracerebral (HIC), reflejado en diversos trabajos como un estudio de casos-control [40] en el que se reclutan hombres y mujeres jóvenes de 44 hospitales de los Estados Unidos para identificar factores de riesgo de la hemorragia intracerebral (HIC) primaria (no asociada con aneurisma, malformación arteriovenosa o lesión estructural); concluyen que los principales factores de riesgo para la HIC primaria pueden ser modificados, y entre éstos se hallan la HTA y el consumo de drogas.

Hasta el momento, no hay grandes estudios que hayan caracterizado la ubicación, la patología, y los resultados en éstos pacientes. En este sentido, este mismo año se ha publicado en Stroke [30], un análisis retrospectivo de 3 años que compara los 45 pacientes ingresados que tenían HIC no traumática y cocaína positiva en orina, con los 105 pacientes similares sin consumo de cocaína. Los autores concluyen que la ingestión reciente de cocaína se asocia con hemorragias que se producen con mayor frecuencia en localización subcortical, tienen un mayor riesgo de hemorragia intraventricular, y tienen un pronóstico peor en comparación con los pacientes con HIC espontánea y cocaínanegativas.

Respecto a la hemorragia subaracnoidea entre consumidores de cocaína se han publicado varios artículos, entre los que destacan los siguientes.

Nanda et al publicaron en 2000 [24] una serie de 14 pacientes con aneurismas relacionados con la cocaína que compararon con 135 pacientes con rotura de aneurisma 
sin historia de consumo de cocaína registrados en el mismo periodo de 6 años. El estudio concluye que el consumo de cocaína predispone a la rotura del aneurisma a una edad significativamente más temprana y en aneurismas de menor tamaño. A diferencia de la literatura publicada, este grupo fue razonablemente bien con un tratamiento agresivo, con pronóstico en cuanto a morbi-mortalidad comparable entre ambos grupos.

El mismo grupo de investigadores ha publicado un artículo reciente en 2008 [23], donde asocian a su serie de 14 pacientes otros 50 de la literatura y los comparan con un grupo control para intentar determinar si el consumo de cocaína es un factor pronóstico significativo entre los pacientes con ruptura de aneurismas intracraneales utilizando las escalas de Hunt y Hess y de Glasgow (GOS); las conclusiones son similares a las previas y en cuanto al grado clínico, no existen diferencias significativas entre los 2 grupos pero el resultado es significativamente peor en los consumidores de cocaína.

Conway y Tamargo publican en Stroke 2001 [26], un artículo en el que analizan la prevalencia de vasoespasmo y resultado clínico en HSA aneurismática asociadas a consumo de cocaína para determinar si la cocaína es un factor de riesgo independiente de vasoespasmo cerebral, concluyendo que sí existe mayor prevalencia de vasoespasmo en los pacientes con consumo reciente de cocaína aunque no existen diferencias en cuanto a los resultados clínicos. Además concluyen que los pacientes son más jóvenes y que la mayoría de los aneurismas son de circulación anterior.

Más tarde, Broderick JP et al en 2003 [39], realizan un estudio de casos y controles de hombres y mujeres jóvenes (menores de 50 años) para identificar factores de riesgo de hemorragia subaracnoidea, demostrando que existe una asociación significativa entre el consumo de cocaína y el riesgo de HSA por rotura de aneurisma; según los autores existen varias hipótesis para explicar la relación entre ambos sucesos, una posibilidad es que las elevaciones agudas de presión arterial asociadas al consumo de cocaína son la causa de ruptura de aneurismas no rotos ya presentes, y la otra posibilidad es, que la cocaína es la responsable del desarrollo de aneurismas y su rotura aguda, debido a la elevación de la presión arterial intracraneal aguda o existencia de arteriopatía. Concluyen que las hemorragias subaracnoideas son situaciones previsibles y prevenibles entre jóvenes y el consumo de cocaína está entre los factores de riesgo más importantes y a su vez modificable.

El resultado de estos artículos [23, 24, 26, 39] sugiere que el consumo de cocaína predispone a la rotura y/o formación de estas alteraciones vasculares, a diferencia de otros trabajos citados previamente [11], donde no se describe ninguna hemorragia 
asociada a aneurisma o malformación AV subyacente. La diferencia en los resultados se debe probablemente a que los ictus isquémicos son atendidos por los neurólogos y, por el contrario, los ictus hemorrágicos son tratados preferentemente por los neurocirujanos, situación que se refleja en los estudios según sean realizados por unos u otros especialistas.

Otros trabajos hacen referencia a la relación de la cocaína y el ictus en situaciones especiales como el de Lucas CE 2005 [28] que establece que el uso de cocaína entre los jóvenes traumatizados en accidentes se asocia a graves efectos adversos fisiopatológicos entre los que se encuentra el ictus, los cuales complican significativamente el diagnóstico inicial y manejo del paciente y consumen gran cantidad de recursos, haciendo imprescindible buscar siempre antecedentes tóxicos entre éstos pacientes; otros autores como WuYW et al 2005 [29] concluyen que el abuso de cocaína entre las madres gestantes que consumieron cocaína durante el embarazo y hasta 15 horas antes del parto, puede ser causa de ictus en recién nacidos y, en el momento del parto especialmente.

Se han utilizado nuevas técnicas de neuroimagen (PET y SPECT) en el estudio de las alteraciones neurotóxicas producidas por la cocaína que aportan más información que las técnicas de imagen estructurales convencionales como la TC y la RM [46].

Por medio de SPECT [19] se ha podido evidenciar alteraciones del flujo sanguíneo cerebral con hipoperfusión en unas zonas e hiperperfusión en otras pudiendo reflejar la acción del vasoespasmo junto a una compensación del flujo sanguíneo cerebral. La rápida reperfusión de áreas previamente isquémicas por el vasoespasmo puede producir una hemorragia. Esta alteración del flujo sanguíneo cerebral puede no producir manifestaciones clínicas detectables. Las alteraciones de perfusión pueden persistir por encima de 6 meses tras la abstinencia en el consumo de cocaína.

Con estas técnicas se ha demostrado hipo-perfusión en corteza occipital y temporal o cerebelo e hiper-perfusión en corteza frontal y parietal en pacientes adictos a cocaína.

Un valioso complemento para las técnica de neuroimagen citadas previamente es la resonancia magnética espectroscópica con la pueden evaluarse cambios neuroquímicos en adictos a cocaína al medir diferentes metabolitos cerebrales como el n-acetilaspartato (marcador neuronal), glutamato (neurotransmisor excitador), creatina y fosfocreatina (metabolismo cerebral) o mioinositol (marcador glial). Un artículo que acaba de publicarse [41] hace una revisión de estudios sobre abuso de drogas que han utilizado esta técnica y han identificado diferentes cambios bioquímicos en el cerebro. Las 
alteraciones más frecuentes con cualquier tóxico es la reducción de $\mathrm{N}$-acetil aspartato y elevación de mioinositol, mientras que los cambios en la colina, creatina, aminoácidos y también son abundantes. Los autores concluyen que el abuso de sustancias tóxicas, puede tener un profundo efecto sobre el metabolismo neuronal que posteriormente podrá trasladarse a los diversos trastornos que éstas ocasionan

El abordaje terapéutico de las complicaciones cerebrovasculares secundarias a la cocaína es similar al utilizado en otras etiologías. Es fundamental el tratamiento de otros factores de riesgo vascular (dislipemia, hipertensión arterial, tabaquismo, diabetes mellitus, obesidad), el tratamiento de patologías concomitantes (arritmias, cardiopatía isquémica, etc.) y el inicio de un tratamiento de la adicción a la cocaína. [8].

Kosten [35], realizó una revisión de cómo el déficit de perfusión cerebral en consumidores de cocaína puede ser un objetivo de la farmacoterapia, destacando entre otros el uso de fármacos que corrijan la disfunción plaquetaria y fármacos neuroprotectores como los antagonistas de glutamato.

En general, se recomienda que el uso de agentes antiagregantes plaquetarios, heparina y anticoagulantes orales en la isquemia vascular cerebral se valore individualmente en cada caso [8].

Según algunos autores [32], aunque los agentes antitrombóticos tienen un potencial en la mitigación de los efectos neurotóxicos de la cocaína, su uso puede estar limitado por el riesgo de hemorragia espontánea.

De nuevo Kosten [42] realiza un estudio para examinar si la reducción del flujo sanguíneo cerebral regional en consumidores de cocaína, se asocia con anormalidades plaquetarias y si estas anormalidades predicen la mejora de hipoperfusión después de 1 mes de abstinencia en pacientes tratados con aspirina frente a placebo. Después de la abstinencia, la hipoperfusión mejora significativamente, independientemente de la asignación del tratamiento, y una mayor agregación plaquetaria al inicio del estudio predice una mayor mejoría en la hipoperfusión. Concluye que la función plaquetaria está relacionada con la hipoperfusión principalmente en el territorio de la arteria cerebral media y que los agentes antiplaquetarios más potentes que la aspirina pueden ser eficaces.

El uso de terapias fibrinolíticas en la isquemia cerebral secundaria a la cocaína no está suficientemente estudiado [8]. Se ha publicado un artículo recientemente en 2009 [10], en el que se realiza una revisión retrospectiva de pacientes con ictus isquémico agudo que recibieron tratamiento trombolítico, comparando un grupo de 29 pacientes 
consumidores de cocaína con otro de 75 pacientes cocaína-negativos. El estudio concluye que los pacientes con los niveles de cocaína positivos y negativos fueron similares en cuanto a la severidad del accidente cerebrovascular y los resultados de seguridad, por lo que la terapia trombolítica parece ser segura en este pequeño estudio, aunque se necesitan más investigaciones para evaluar de manera más definitiva la seguridad y la eficacia de este tratamiento.

Revisando la patogenia de la isquemia cerebral relacionada con la cocaína y posibles agentes terapéuticos, diversos autores han evaluado el papel de los antagonistas dihidropiridínicos de los canales de calcio. El motivo de su utilización se basa en el hecho de que el vasoespasmo arterial cerebral inducido por la cocaína es consecuencia de los niveles aumentados de monoaminas, en especial de dopamina.

Johnson et al [32] en 2001, realizan una revisión de la literatura de 1966 y 2000, y evalúan el papel de los supuestos agentes terapéuticos, en particular, de los antagonistas de los canales de calcio dihidropiridínicos tales como isradipina, y concluyen que éstos, al inhibir la liberación de dopamina en las neuronas, son una promesa como agentes terapéuticos para la prevención de la isquemia cerebral inducida por cocaína.

Años más tarde, estos mismos investigadores, llevan a cabo un ensayo clínico [33], con sujetos adictos a cocaína y anfetaminas, a los que antes de la administración intravenosa de los tóxicos, tratan con isradipina vs placebo, comprobando que este fármaco reduce significativamente el aumento de la presión arterial asociado al tóxico pero con tendencia a aumentar la frecuencia cardíaca; concluyen que son necesarios más estudios clínicos para determinar si isradipina es terapéuticamente eficaz en la prevención de las crisis hipertensivas y las secuelas cerebrovasculares y cardiovasculares asociadas al consumo de cocaína o metanfetamina. Puesto que hasta el momento no existe tratamiento farmacológico específico establecido, la identificación de un fármaco eficaz sería científica y clínicamente importante.

En resumen, el uso de fármacos antagonistas del calcio permanece contradictorio, pero no están contraindicados.

De manera sintomática, la vasoconstricción producida por la cocaína y la hipertensión arterial secundaria a la misma se tratan con benzodiacepinas inicialmente. Se puede utilizar fentolamina, un antagonista alfa-adrenérgico, a dosis de $1 \mathrm{mg}$ intravenosos, repetible a los 5 minutos o en infusión a $0.4 \mathrm{mg} /$ minuto. La nitroglicerina y el nitroprusiato sódico en perfusión pueden ser también utilizados ante cuadros de hipertensión arterial severos [8]. 
No se deben utilizar betabloqueantes en el tratamiento de las complicaciones cardiovasculares secundarias a la cocaína. El efecto beta antagonista deja sin oposición el efecto alfa agonista de la cocaína, por lo que aumentará la vasoconstricción y la presión arterial, con los consiguientes efectos deletéreos sobre la perfusión de los tejidos. A pesar de que el labetalol tenga un actividad mixta beta y alfa, predomina el antagonismo beta y no se recomienda su utilización.

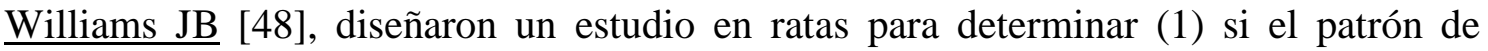
respuesta hemodinámica (vascular o mixta) a la cocaína se correlaciona con la sensibilidad relativa a la toxicidad y (2) si alterar el patrón de respuesta hemodinámica a la cocaína utilizando el propranolol aumenta la toxicidad. Tras realizar el ensayo, los autores proponen que el modelo inicial de la respuesta hemodinámica a la cocaína predice la sensibilidad a la toxicidad de la cocaína y que el propranolol, un fármaco que mejora el aumento de la resistencia vascular a la cocaína, también aumenta la toxicidad de la cocaína en respuesta vascular.

Como curiosidad, existe un caso descrito en la literatura [37] de tromboaspiración y recanalización de la arteria basilar en un joven en coma tras oclusión aguda de dicha arteria asociada al uso de cocaína y éxtasis; la recuperación del paciente fue favorable y se cita a la tromboaspiración como posible terapia alternativa a la trombolisis.

\section{$\checkmark$ Crisis epilépticas}

Las convulsiones son una complicación frecuente en la intoxicación por sustancias simpaticomiméticas como la cocaína. Aunque el mecanismo fisiopatológico no está aclarado completamente, se relaciona con el bloqueo de la recaptación de catecolaminas.

Predominan las crisis comiciales generalizadas tónico-clónicas, pero también se han descrito crisis parciales simples y complejas. Puede aparecer un estatus epiléptico en pacientes con consumo de una alta dosis de cocaína, incluso sin antecedentes de epilepsia.

La aparición de una crisis convulsiva tras el consumo de cocaína puede suceder incluso varias horas tras el consumo según la vía de administración. Hay que valorar el consumo o la deprivación de otros tóxicos con capacidad de provocar convulsiones como las anfetaminas, éxtasis, cannabis y alcohol. Ante una crisis convulsiva atribuida al consumo de cocaína se debe valorar la posibilidad de ser secundaria a un accidente cerebrovascular tanto de tipo isquémico como hemorrágico. 
El tratamiento es el de una crisis convulsiva sin importar la etiología. No está demostrada la necesidad de continuar con tratamiento antiepiléptico convencional en casos de crisis convulsivas por cocaína, aunque se debe individualizar cada caso [8].

\section{Leucoencefalopatía por cocaína}

La leucoencefalopatía es una lesión estructural de la sustancia blanca en la cual la mielina se afecta de forma preponderante. Existen diferentes etiologías entre las que se encuentran los tóxicos. En el caso de la cocaína, está menos dilucidado que con la heroína. Las manifestaciones clínicas pueden muy diversas.

La RM es la técnica de elección para evaluar la sustancia blanca. Se ha visto que en sujetos consumidores de cocaína, asintomáticos, presentan más alteraciones de la sustancia blanca en RNM que en sujetos control no consumidores, sugiriéndose que estos sujetos presentan un riesgo mayor de isquemia inducida por vasoespasmo en la sustancia blanca.

El tratamiento se centra en la abstinencia en el consumo de estas drogas de abuso con capacidad leucotóxica, habiéndose descrito mejorías clínicas y radiológicas con dicha abstinencia [8].

\section{Cefalea}

El tipo de cefaleas que más frecuentemente se presentan entre los usuarios de cocaína es de tipo migrañoso [51]. La prevalencia de las cefaleas entre los consumidores de cocaína difiere entre los diversos estudios. Se han identificado diferentes patrones de presentación de cefaleas $[6,8]$ :

- cefalea aguda tras el consumo de cocaína con una duración entre 2 y 48 horas, predominio occipital y pulsátil, asociada con fotofobia, nauseas, vómitos.

- cefalea de intensidad progresiva durante un episodio de atracón o "binge", con duración de varios días, predominio frontal, pulsátil y acompañada de mareos, náuseas y vómitos.

- cefaleas durante periodos de abstinencia. Ocurre de 1 a 4 días tras el último consumo y empeora en los días sucesivos, con predominio frontal, pulsátil, acompañada de fotofobia, náuseas y vómitos.

- También las cefaleas en racimos o "cluster headache" [50]

La cefalea producida por la cocaína está en relación con su acción sobre el sistema serotoninérgico, debido al bloqueo en la recaptación de serotonina [9, 6]. Algunos 
autores [51] plantean que las cefaleas agudas tras el consumo de cocaína pueden estar relacionadas con los efectos simpaticomiméticos o vasoconstrictoras de la cocaína, mientras que cefalea tras la retirada de cocaína o durante un "atracón" puede relacionarse con la alteración inducida por cocaína del sistema serotoninérgico.

Ante la aparición de un cuadro de cefaleas en relación con el consumo de cocaína lo primordial es realizar un diagnóstico diferencial completo que excluya otras causas de cefalea relacionada o no con el consumo de cocaína (ulceración tabique nasal-sinusitis, crisis hipertensiva, accidente vascular cerebral) [8].

El tratamiento de esta cefalea se realiza con fármacos antiinflamatorios no esteroideos y ergotamínicos por su acción serotoninérgica.

\section{$\checkmark$ Rabdomiolisis}

Se han publicado diversos artículos que realacionan el consumo de cocaína con rabdomiolisis aguda. En su aparición intervienen diferentes factores como la hipertermia y sobreestimulación simpática, la agitación, las crisis convulsivas, la vasoconstricción arterial que produce isquemia muscular o por acción tóxica directa de la cocaína en el metabolismo muscular [8]. En éstos se describe la presencia de rabdomiolisis con fallo renal tras el uso intranasal de cocaína, que cursa con incremento de creatin fosfokinasa. Se ha visto que en pacientes consumidores de cocaína que se encuentran asintomáticos presentan niveles elevados de creatinfosfoquinasa.

El tratamiento es el propio de la rabdomiolisis por cualquier otra causa.

\section{$\checkmark$ Alteraciones neuropsicológicas.}

Se han descrito alteraciones neuropsicológicas en los adictos a la cocaína que abarcan desde alteraciones del humor, anhedonia y síntomas depresivos que se relacionan con alteraciones en los receptores de catecolaminas por la acción de la cocaína de forma crónica [8].

En este campo se publicó un trabajo en 2007 [22], en el que utilizan las técnicas de neuroimagen para estudiar la relación entre el consumo de drogas y las alteraciones neuropsicológicas vinculadas al funcionamiento de diversas regiones cerebrales. Los hallazgos en consumidores de cocaína revelan alteraciones significativas y estables en diversas regiones dentro del córtex prefrontal, en la sustancia blanca subyacente y en una serie de estructuras subcorticales que incluyen las ínsulas, la amígdala y los ganglios basales. En consonancia, los estudios de imagen funcional han demostrado que 
estas mismas regiones muestran una actividad reducida en respuesta a las demandas de tareas de memoria operativa, inhibición y toma de decisiones. También se han observado correlaciones significativas entre el metabolismo basal de las áreas prefrontales y el rendimiento en tareas de función ejecutiva. Por tanto, las alteraciones observadas en estos estudios pueden contribuir a explicar los déficit cognitivos y motivacionales de los consumidores de cocaína. 


\section{OBJETIVOS}

\section{Principal:}

1. Analizar la implicación de la cocaína en la patología vascular cerebral en pacientes jóvenes.

2. Describir y analizar la morbi-mortalidad de este grupo etiológico frente a un grupo control para intentar hallar la relevancia del consumo de este tóxico como factor de riesgo cerebrovascular en pacientes jóvenes sin otros FRCV conocidos.

\section{Secundarios:}

1. Descripción de las características demográficas y factores de riesgo de los pacientes y compararla con el grupo control.

2. Descripción del tipo de lesiones y su localización comparándolas con el grupo control.

3. Descripción de alteraciones detectadas en las pruebas complementarias realizadas.

4. Descripción de la evolución: morbimortalidad y pronóstico neurológico y funcional al alta determinado por las escalas de la NIH y Rankin modificada y compararlo con el grupo control. 


\section{MATERIAL Y MÉTODOS}

Se realiza una revisión retrospectiva de pacientes menores de 50 años ingresados en el servicio de Neurología de nuestro hospital desde Enero 2006 hasta Diciembre 2009. Se obtienen 32 pacientes con niveles de cocaína positivos al ingreso, y de éstos se seleccionan 18 pacientes en los el consumo de cocaína se asocia a ictus isquémico y hemorrágico.

Por otra parte, se revisan los pacientes menores de 50 años, ingresados en la Unidad de Ictus de nuestro servicio en ese mismo periodo, con niveles de cocaína negativos al ingreso; se obtiene un total de 100 pacientes, y de éstos se selecciona 79 pacientes con diagnóstico final de ictus isquémico y hemorrágico. Estos pacientes constituirán el grupo control en nuestro estudio.

Con el total de 132 pacientes se crea una base de datos en el programa informático PASW Statistics18, estableciendo un total de 63 variables, de las cuales se analizarán 57. En éstas se recoge información sobre datos del paciente, demográficos, estancia hospitalaria, consumo de tóxicos, antecedente médicos, factores de riesgo cardiovascular, pruebas complementarias, diagnósticos, complicaciones y morbimortalidad.

Finalmente, se realiza un análisis estadístico descriptivo de los 97 pacientes que forman nuestra población (18 cocaína positivos y 79 cocaína negativos) y se comparan los resultados entre sí y con los encontrados en la literatura mediante la revisión bibliográfica que se ha llevado a cabo previamente. Para completar el trabajo, se decide realizar un análisis univariante con diversas pruebas no paramétricas de alguna de las variables que consideramos de interés.

\section{SELECCIÓN DE PACIENTES}

Los pacientes incluidos en el estudio deben cumplir todos los criterios de inclusión y ninguno de los criterios excluyentes:

\section{* Criterios de inclusión:}

- Pacientes menores de 50 años ingresados en el Servicio de Neurología con patología cerebrovascular aguda.

- Niveles de cocaína positivo en orina al ingreso. 


\section{* Criterios de exclusión:}

- No cumplir criterios de inclusión.

Al tratarse de un estudio retrospectivo, debemos asumir que en la selección de pacientes, puede existir un sesgo de información, ya que se incluye en el grupo de no consumidores de cocaína, a todos aquellos pacientes de los que no se tienen nniveles de cocaína positivo en orina al ingreso, sin poder asegurar que no la tuvieran ya que en algunos casos carecemos de esta información.

\section{SELECCIÓN DE VARIABLES}

Los datos recogidos son:

- Datos del paciente, con número de identificación asignado para mantener la confidencialidad.

- Datos demográficos: edad, sexo.

- Días de estancia en el hospital.

- Consumo de tóxicos distintos de cocaína: tabaco, alcohol, cannabis, opiáceos, drogas de diseño.

- Antecedente médicos: cefalea, patología psiquiátrica, virus hepatitis o VIH, ictus.

- Factores de riesgo cardiovascular: dislipemia, diabetes, HTA.

- Datos analíticos: CPK, ácido fólico, considerándose patológicos:

○ $\mathrm{CPK}>145 \mathrm{U} / \mathrm{L}$

○ Acido Fólico $<2.7 \mathrm{ng} / \mathrm{ml}$

- Datos de pruebas complementarias:

○ RM Cerebral: determinando la presencia y topografía de la lesión.

- Estudio vascular (Angio-RM/Angio-TC/Doppler/Dupplex/Arteriografía): determinando la presencia o ausencia de aneurismas, malformaciones arteriovenosas, estenosis u oclusión arterial, disección arterial o vasculitis).

- Estudio cardiológico con ECG y Ecocardiograma: determinar presencia de cardiopatías embolígenas (cardiopatía isquémica, miocardiopatía dilatada, valvulopatía, shunt derecha-izquierda).

○ Estudio de trombosis.

- Datos diagnósticos: motivo de ingreso, diagnóstico clínico, topográfico y etiológico. 
- Datos de complicaciones neurológicas o sistémicas durante el ingreso. Datos de morbi-mortalidad: presencia o ausencia de secuelas, escala modificada de Rankin y $\mathrm{NIH}$ al ingreso y al alta.

\section{DEFINICIÓN DE VARIABLES}

En la definición de las 57 variables seleccionadas, se establecen los siguientes valores o categorías:

- Las variables de edad y estancia hospitalaria se dividen en 2; edad exacta y rango de edad, estableciendo 3 categorías (<20 años, 20-40 años, > 40 años) y días de estancia exactos y rango de días, estableciendo 3 categorías (< 7 días, 7-14 días, > 14 días).

- Se crean 5 variables de consumo de otros tóxicos (tabaco, alcohol, cannabis, opiáceos y drogas de síntesis); para cada una de éstas variables se se establecen los valores sí o no.

- En cuanto a los antecedentes médicos, se diferencian 5 variables (cefalea, patología psiquiátrica, virus hepatitis o VIH, ictus y otros); para cada una de éstas variables se se establecen los valores sí o no.

- Para los factores de riesgo cardiovascular se establecen en primer lugar 2 variables (FRCV al ingreso y FRCV al alta) y en segundo lugar 3 variables con cada uno de los FRCV recogidos (dislipemia, DM, HTA) al ingreso y otras 3 con los FRCV al alta; para todas estas variables se establecen los valores sí o no.

- Para las vvariables de datos analíticos y pruebas complementarias se realiza la siguiente distribución:

○ Hipercekaemia y déficit de ácido fólico se catalogan como sí o no.

○ RM Cerebral: realmente se trata de dos variables; la primera de ellas es realización de RM y por tanto las categorías son sí o no, la segunda es resultado de RM, y las categorías son normal o patológica (ictus isquémico/hemorrágico).

○ Estudio vascular (Angio-RM/Angio-TC/Doppler/Dupplex/Arteriografía): en primer lugar se establecen 2 variables; a/ realización de estudio vascular, con categorías sí o no y b/ resultado de estudio vascular con las siguientes categorías: normal, estenosis ACI $<50 \%$, estenosis ACI > 50\%, oclusión ACI, estenosis en tándem, estenosis intracraneal, estenosis vertebrobasilar, aneurismas cerebral, malformaciones arteriovenosas y otros hallazgos. En segundo lugar, se establece 
una variable independiente que será disección arterial, cuyas categorías son sí o no.

○ Estudio cardiológico:

- ECG: se establecen 2 variables; a/ realización de ECG, con categorías sí o no y b/ hallazgo de FA, con categorías sí o no.

- Ecocardiograma: en primer lugar se establecen 2 variables; a/ realización de ecocardiograma, con categorías sí o no y b/ resultado de ecocardiograma con las categorías normal o patológico. En segundo lugar, se establecen cinco variables independientes que serán cardiopatía isquémica, miocardiopatía dilatada, valvulopatía, shunt derecha-izquierda e HVI.

○ Estudio de trombosis: se divide en dos variables; la primera de ellas es realización del estudio, con las categorías sí o no y la segunda es resultado del estudio, con las categorías normal o patológico.

- Las variables de diagnóstico, se clasifican de la siguiente manera:

○ Motivo de ingreso: se establecen tres categorías, ictus isquémico, hemorrágico y HSA.

- Diagnóstico clínico: se establecen seis categorías, de las cuales dos son para las hemorragias, 1/HEMO (hemorragia intracerebral), 2/HSA (hemorragia subaracnoidea) y las cuatro restantes para los ictus isquémicos según la clasificación OCSP (Oxfordshire Community Stroke Project), 3/TACI (infarto total de la circulación anterior), 4/PACI (infarto parcial de la circulación anterior), 5/LACI (infarto lacunar), 6/POCI (infarto de la circulación posterior).

○ Diagnóstico topográfico 1: se diferencian 6 categorías en función de la arteria cerebral afectada (ACA, ACM, ACP, VB, carotídeo, indeterminado).

○ Diagnóstico topográfico 2: se diferencian 3 categorías según la lesión sea cortical, profunda o ambos.

- Diagnóstico etiológico: se diferencian 12 categorías, 1/ AIT, 2/ Ictus establecido: Hemorragia cerebelosa, 3/ Ictus establecido: Hemorragia intraparenquimatosa, 4/ Ictus establecido: Hemorragia intraventricular, 5/ Ictus establecido: Hemorragia subaracnoidea, 6/ Ictus establecido: Infarto cerebral aterotrombótico, 7/ Ictus establecido: Infarto cerebral cardioembólico, 8/ Ictus establecido: Infarto cerebral hemorrágico, 9/ Ictus establecido: Infarto cerebral lacunar, 10/ Ictus establecido: Infarto cerebral de causa inhabitual, 11/ Ictus establecido: Infarto cerebral indeterminado. 
- Las complicaciones se dividen en tres variables diferentes:

○ Existencia complicaciones: se establecen 4 categorías, complicaciones neurológicas, cardiovasculares, sistémicas o ninguna.

○ Complicaciones neurológicas: se establecen 14 categorías, 1/ crisis epilépticas focales, 2/ crisis epilépticas focal $2^{\mathrm{a}}$ generalizada, 3/ crisis epilépticas generalizadas, 4/ crisis epilépticas: status, 5/ edema cerebral, 6/ extensión del hematoma, 7/ hidrocefalia, 8/ transformación hemorrágica no sintomática, 9/ transformación hemorrágica sintomática, 10/ vasoespasmo, 11/ progresión del ictus, 12/ recurrencia, 13/ninguna, 14/otras.

○ Complicaciones sistémicas: se establecen 8 categorías, 1/ fiebre, 2/ ITU, 3/ neumonía, 4/ infecciones de catéter venoso, 5/ sepsis, 6/ hemorragia sistémica mayor, $7 /$ rabdomiolisis, $8 /$ otras, $9 /$ ninguna.

- En cuanto a la morbimortalidad se crean 6 variables diferentes:

- Secuelas neurológicas: estableciendo 3 categorías, con secuelas neurológicas, sin secuelas neurológicas y éxitus.

- Tipo de secuela neurológica: se establecen 6 categorías, 1/ encefalopatía hipóxica, 2/ déficit motor, 3/ déficit sensitivo, 4/ trastorno del lenguaje, 5/ varios, 6/ otras.

○ Rankin al ingreso: las categorías son la propia escala de Rankin modificada, donde 0 es asintomático y 6 es éxitus.

○ Rankin al alta: similar a la previa pero con la puntuación del paciente al alta en función de las secuelas.

- NIHSS al ingreso: las categorías son las propias puntuaciones de la escala en la primera exploración neurológica que se realiza al ingreso.

- NIHSS al alta: similar a la previa, pero la última exploración que se realiza previa al alta hospitalaria; los pacientes que fallecen, obtienen la puntuación máxima (42).

\section{ANÁLISIS ESTADÍSTICO REALIZADO}

Se realiza un análisis descriptivo de ambas muestras, en el cual, las variables cuantitativas (edad y estancia media) se expresan en forma de media \pm desviación estándar, mientras que las variables cualitativas se expresan en porcentajes.

El estudio estadístico se amplía tratando de valorar si existen diferencias estadísticamente significativas entre ambas poblaciones en cuanto a edad, estancia 
hospitalaria, factores de riesgo (HTA), morbilidad y mortalidad. Para ello se realiza un análisis univariante en el que se emplean las pruebas no paramétricas correspondientes. Para comparar ambas muestras entre variables categóricas se utiliza la prueba X2 de Pearson. La comparación con variables cuantitativas se realiza mediante el test de la U de Mann-Whitney. Consideramos estadísticamente signifativo un valor de $\mathrm{p}$ inferior a 0,05 . 


\section{RESULTADOS}

\section{ANÁLISIS DESCRIPTIVO}

\section{Datos demográficos}

La distribución por sexos del total de la muestra es de 61 varones (62.9\%) y 36 mujeres $(37.1 \%)$; en el grupo consumidor de cocaína: 10 varones $(55.6 \%)$ y 8 mujeres (44.4\%); y en el grupo control: 51 varones (64.6\%) y 28 mujeres (35.4\%).

La edad media del total de la muestra es de $40.36 \pm 8.30$; en el grupo consumidor de cocaína 35.22 años \pm 8.99 y un rango de (18-50) y en el grupo control 41.53 años \pm 7.73 con un rango de (16-50).

\section{Datos de estancia hospitalaria:}

La estancia hospitalaria media del total de la muestra es de $9.26 \pm 6.67$; en el grupo consumidor de cocaína 9.28 días \pm 5.65 con un rango de (2-24) y en el grupo control 9.25 días \pm 6.91 con un rango de (2-36).

\section{Distribución de consumo de otros tóxicos:}

- Tabaco: 59 (60.8\%) de los 97 pacientes eran fumadores; en el grupo consumidor de cocaína 15 de los 18 pacientes (83.3\%) y en el grupo control 44 de los 79 pacientes $(55.7 \%)$.

- Alcohol: 39 (40.2\%) de los 97 pacientes consumían alcohol; en el grupo consumidor de cocaína 14 de los 18 pacientes $(77.8 \%)$ y en el grupo control 25 de los 79 pacientes $(31.6 \%)$.

- Cannabis: 9 (9.3\%) de los 97 pacientes consumían cannabis; en el grupo consumidor de cocaína 7 de los 18 pacientes (38.9\%) y en el grupo control 2 de los 79 pacientes (2.5\%).

- Opiáceos: sólo 1 paciente (1\%) del total, perteneciente al grupo control era consumidor de opiáceos.

- Drogas de síntesis: sólo 2 pacientes $(2.1 \%)$ del total, pertenecientes al grupo consumidor de cocaína, lo eran también de drogas de síntesis. 


\section{Distribución de los antecedentes médicos}

- Ictus previo: sólo 5 pacientes (6.3\%) del total, habían presentado previamente un ictus, todos ellos pertenecientes al grupo control.

- Cefalea: existía antecedente de cefalea en 22 (22.7\%) del total de pacientes; en el grupo consumidor de cocaína 3 de los 18 pacientes (16.7\%) y en el grupo control 19 de los 79 pacientes $(24.1 \%)$.

- Trastorno psiquiátricos: existía antecedente de trastorno psiquiátrico (trastorno del ánimo) en $6(6.2 \%)$ del total de pacientes; en el grupo consumidor de cocaína 3 de los 18 pacientes $(16.7 \%)$ y en el grupo control 3 de los 79 pacientes $(3.8 \%)$.

- Enfermedades víricas (VHB, VHC, VIH): existía antecedente de enfermedad vírica en 7 (7.2\%) del total de pacientes; en el grupo consumidor de cocaína 3 de los 18 pacientes (16.7\%) y en el grupo control 4 de los 79 pacientes $(5.1 \%)$.

Distribución de los factores de riesgo cardiovascular al ingreso

- Factores de riesgo cardiovascular al ingreso (HTA, DL, DM): 32 pacientes del total $(33 \%)$, presentaban antecedentes de FRCV; en el grupo consumidor de cocaína 3 de los 18 pacientes (16.7\%) y en el grupo control 29 de los 79 pacientes $(36.7 \%)$.

- Dislipemia: 15 pacientes del total (15.5\%), presentaban antecedentes de dislipemia; en el grupo consumidor de cocaína 2 de los 18 pacientes $(11.1 \%)$ y en el grupo control 13 de los 79 pacientes (16.5\%).

- Diabetes Mellitus: 8 pacientes del total (8.2\%), presentaban antecedentes de diabetes mellitus; en el grupo consumidor de cocaína 1 de los 18 pacientes (5.6\%) y en el grupo control 7 de los 79 pacientes (8.9\%).

- HTA: 22 pacientes del total (22.7\%), presentaban antecedentes de HTA; en el grupo consumidor de cocaína 2 de los 18 pacientes (11.1\%) y en el grupo control 20 de los 79 pacientes $(25.3 \%)$.

\section{Distribución de los factores de riesgo al alta}

- Factores de riesgo cardiovascular al alta (HTA, DL, DM): 55 pacientes del total (56.7\%), presentaban FRCV al alta; en el grupo consumidor de cocaína 7 de los 18 pacientes (38.9\%) y en el grupo control 48 de los 79 pacientes $(60.8 \%)$.

- Dislipemia: 43 pacientes del total (44.3\%), presentaban dislipemia al alta; en el grupo consumidor de cocaína 7 de los 18 pacientes (38.9\%) y en el grupo control 36 de los 79 pacientes $(45.6 \%)$. 
- Diabetes Mellitus: 11 pacientes del total (11.3\%), presentaban diabetes mellitus al alta; en el grupo consumidor de cocaína 1 de los 18 pacientes (5.6\%) y en el grupo control 10 de los 79 pacientes (12.7\%).

- HTA: 26 pacientes del total (26.8\%), presentaban HTA al alta; en el grupo consumidor de cocaína 4 de los 18 pacientes (22.2\%) y en el grupo control 22 de los 79 pacientes $(27.8 \%)$.

\section{Distribución de datos analíticos.}

- Hipercekaemia: 9 pacientes del total (9.3\%) presentó elevación de CK; en el grupo consumidor de cocaína 5 de los 18 pacientes (27.8\%) y en el grupo control 4 de los 79 pacientes $(5.1 \%)$.

- Déficit de Acido Fólico: 12 pacientes del total (12.4\%) presentó déficit de ácido fólico; en el grupo consumidor de cocaína 3 de los 18 pacientes (16.7\%) y en el grupo control 9 de los 79 pacientes $(11.4 \%)$.

\section{Distribución del estudio de neuroimagen:}

- RM Cerebral se realizó a 80 pacientes del total (82.5\%), de los cuales 13 pertenecen al grupo consumidor de cocaína (72.2\%) y 67 al grupo control (84.8\%).

- El resultado de la RM Cerebral fue normal en 14 pacientes del total, de los cuales 2 pertenecen al grupo consumidor de cocaína (11.1\%) y 12 al grupo control (15.2\%).

\section{Características del estudio vascular cerebral}

- Estudio vascular (Angio-RM/Angio-TC/Doppler/Dupplex/Arteriografía) se realizó a 93 pacientes del total $(95.9 \%)$, de los cuales 17 pertenecen al grupo consumidor de cocaína $(94.4 \%)$ y 76 al grupo control $(96.2 \%)$.

- El resultado del estudio vascular fue normal en 61 pacientes del total (62.9\%), con hallazgo de estenosis ACI $<50 \%$ en $1(1 \%)$, estenosis ACI $>50 \%$ en $1(1 \%)$, oclusión ACI en 3 (3.1\%), estenosis en tándem en 2 (2.1\%), estenosis intracraneal en $11(11.3 \%)$, estenosis vertebrobasilar en 2 (2.1\%), aneurismas cerebral en 6 (6.2\%), malformaciones arteriovenosas en $1(1 \%)$ y otros hallazgos en $5(5.2 \%)$. De todas estas alteraciones, en el grupo consumidor de cocaína, sólo se encontró estenosis intracraneal en $2(11.1 \%)$ y aneurismas cerebral en $2(11.1 \%)$; es decir, 
que en este grupo, el estudio vascular fue normal en 13 pacientes $(72.2 \%)$ frente a 48 pacientes $(60.8 \%)$ del grupo control.

- Se diagnosticó disección arterial en 5 pacientes del total (5.2\%), pertenecientes todos ellos al grupo control.

\section{Descripción del estudio cardiológico.}

- ECG se realizó a todos los pacientes con hallazgo de arritmia por FA en 6 (6.2\%) del total correspondiendo todos ellos al grupo control.

- Ecocardiograma se realizó a 68 pacientes del total de la muestra (70.1\%), 10 (55.6\%) del grupo consumidor de cocaína y 58 (73.4\%) del grupo control. El resultado fue normal en 53 pacientes del total (54.6\%) y patológico en 15 (15.5\%) de los cuales $2(11.1 \%)$ pertenecen al grupo de cocaína y $13(16.5 \%)$ al grupo control. Los hallazgos encontrados fueron de miocardiopatía dilatada en 3 (3.1\%), HVI en $8(8.2 \%)$, cardiopatía isquémica en $4(4.1 \%)$ y valvulopatía en 7 (7.2\%); todos éstos, pertenecen al grupo control excepto 1 caso de HVI (5.6\%) y un caso de valvulopatía (5.6\%) que pertenecen al grupo de cocaína. Por otra parte se diagnosticó shunt derecha-izquierda en 11 pacientes (11.3\%), 1 (5.6\%) del grupo consumidor de cocaína y $10(12.7 \%)$ del grupo control.

\section{Descripción del estudio de trombosis}

- El estudio de trombosis se realizó en 63 pacientes (64.9\%), 11 (61.1\%) del grupo de cocaína y 52 (65.8\%) del grupo control y el resultado del estudio fue patológico en 8 pacientes $(8.2 \%)$, de los cuales sólo 1 paciente $(5.6 \%)$ pertenece al grupo consumidor de cocaína y 7 (8.9\%) al grupo control.

\section{Descripción de los diagnósticos}

- El motivo de ingreso para el total de pacientes fue de ictus isquémico en 71 (73.2\%), hemorrágico en $12(12.4 \%)$ y HSA en 12 (12.4\%); en el grupo consumidor de cocaína, ictus isquémico en $11(61.1 \%)$, hemorrágico en $2(11.1 \%)$ y HSA en 3 (16.7\%); y en el grupo control ictus isquémico en 60 (75.9\%), hemorrágico en 10 (12.7\%) y HSA en 9 (11.4\%). 
- Diagnóstico clínico: 15 pacientes del total (15.5\%) presentan hemorragia intracerebral, $12(12.4 \%)$ hemorragia subaracnoidea, $13(13.4 \%)$ TACI (infarto total de la circulación anterior), 15 (15.5\%) PACI (infarto parcial de la circulación anterior), 28 (28.9\%) LACI (infarto lacunar), 14 (14.4\%) POCI (infarto de la circulación posterior); en el grupo consumidor de cocaína 4 (22.2\%) hemorragia intracerebral, 3 (16.7\%) hemorragia subaracnoidea, 4 (22.2\%) TACI, 7 (38.9\%) LACI y ningún PACI ni POCI; y en el grupo control 11 (13.9\%) presentan hemorragia intracerebral, $9(11.4 \%)$ hemorragia subaracnoidea, 9 (11.4\%) TACI, 15 (19\%) PACI, 21 (26.6\%) LACI, 14 (17.7\%) POCI.
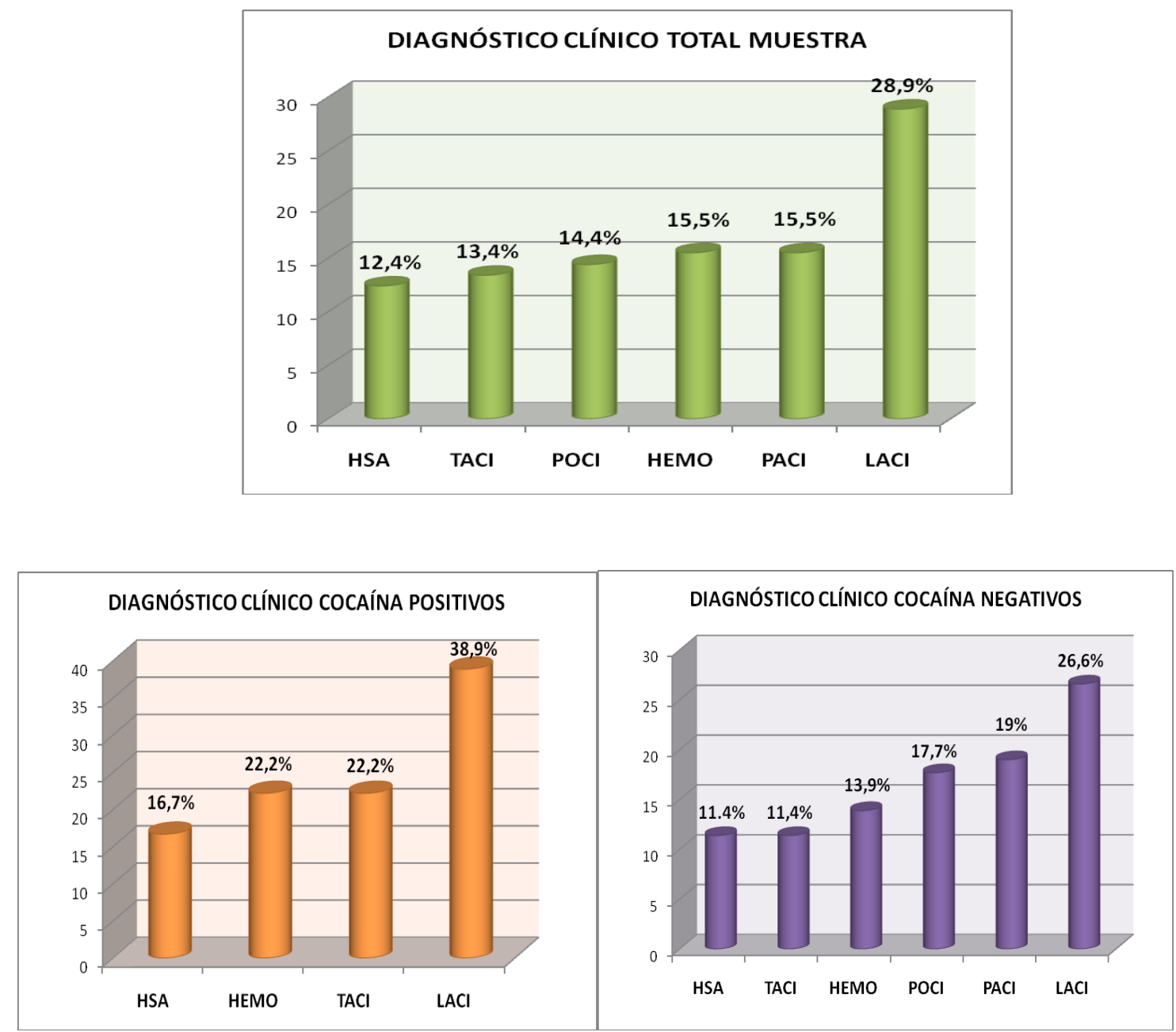

- Diagnóstico topográfico 1: del total de pacientes, la arteria cerebral afectada fue ACA en 3 (3.1\%), ACM en 52 (53.6\%), ACP en 7 (7.2\%), VB en 19 (19.6\%), carotídeo en $11(11.3 \%)$, indeterminado en 5 (5.1\%); en el grupo consumidor de cocaína ACA en 1 (5.6\%), ACM en 11 (61.1\%), ACP en 2 (11.1\%), VB en 1 (5.6\%), 
carotídeo en 1 (5.6\%), indeterminado en 2 (11.1\%); en el grupo control ACA en 2 (2.5\%), ACM en 41 (51.91\%), ACP en 5 (6.3\%), VB en 18 (22.8\%), carotídeo en 10 (12.7\%). Por tanto el territorio arterial comprometido en el grupo de cocaína, correspondía a la circulación anterior en $72.3 \%$ y a la posterior en $16.7 \%$.
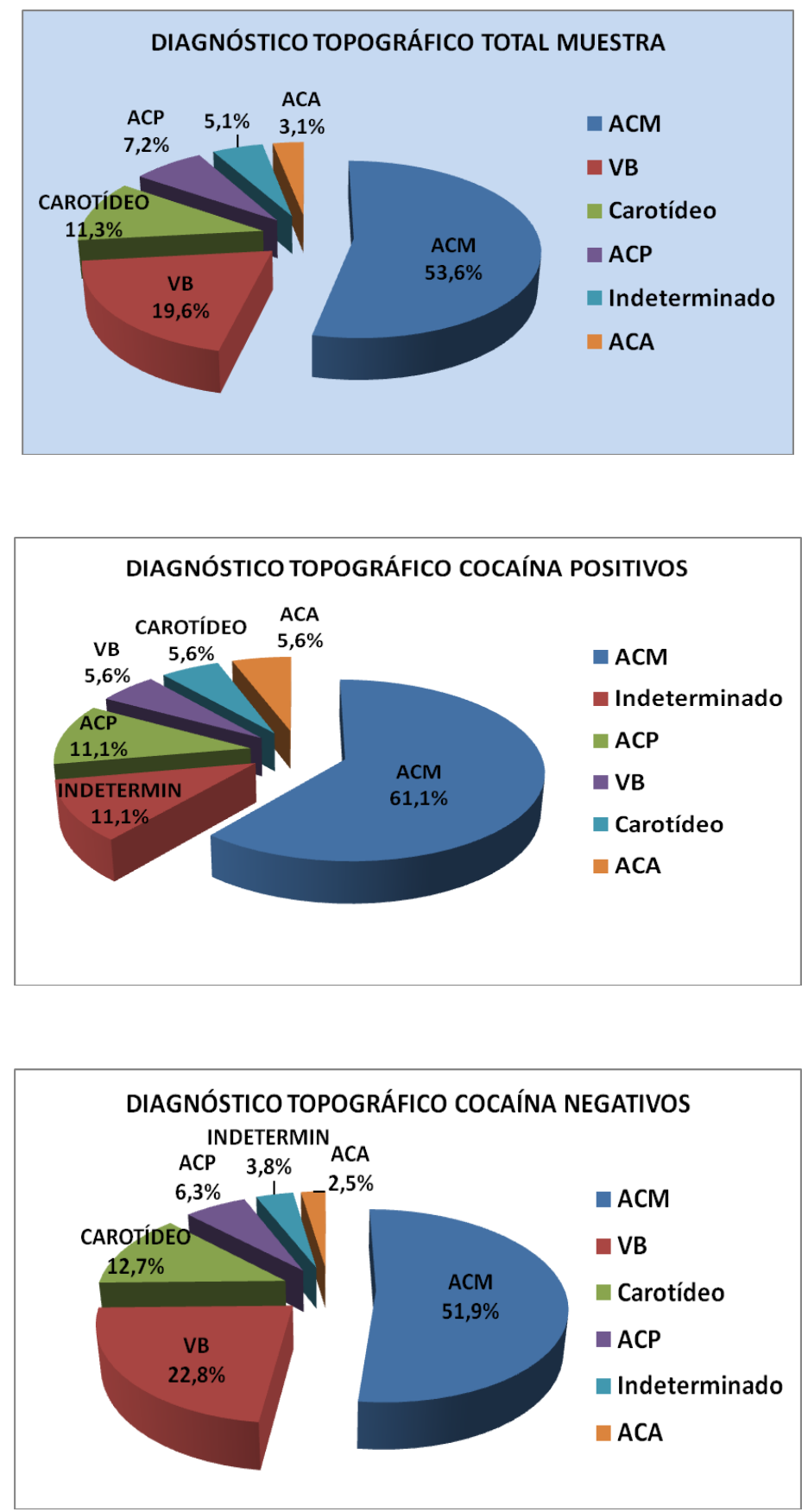

- Diagnóstico topográfico 2 (esta variable sólo se establece en 46 pacientes): del total de pacientes la lesión se localizó en territorio cortical en 13 (13.4\%), profunda en 25 (25.8\%) y ambos en 13 (13.4\%); en el grupo consumidor de cocaína, cortical en 2 
(11.1\%), profunda en 5 (27.8\%) y ambos en 4 (22.2\%); en el grupo control, cortical en $11(13.9 \%)$, profunda en $20(25.3 \%)$ y ambos en $9(11.4 \%)$.
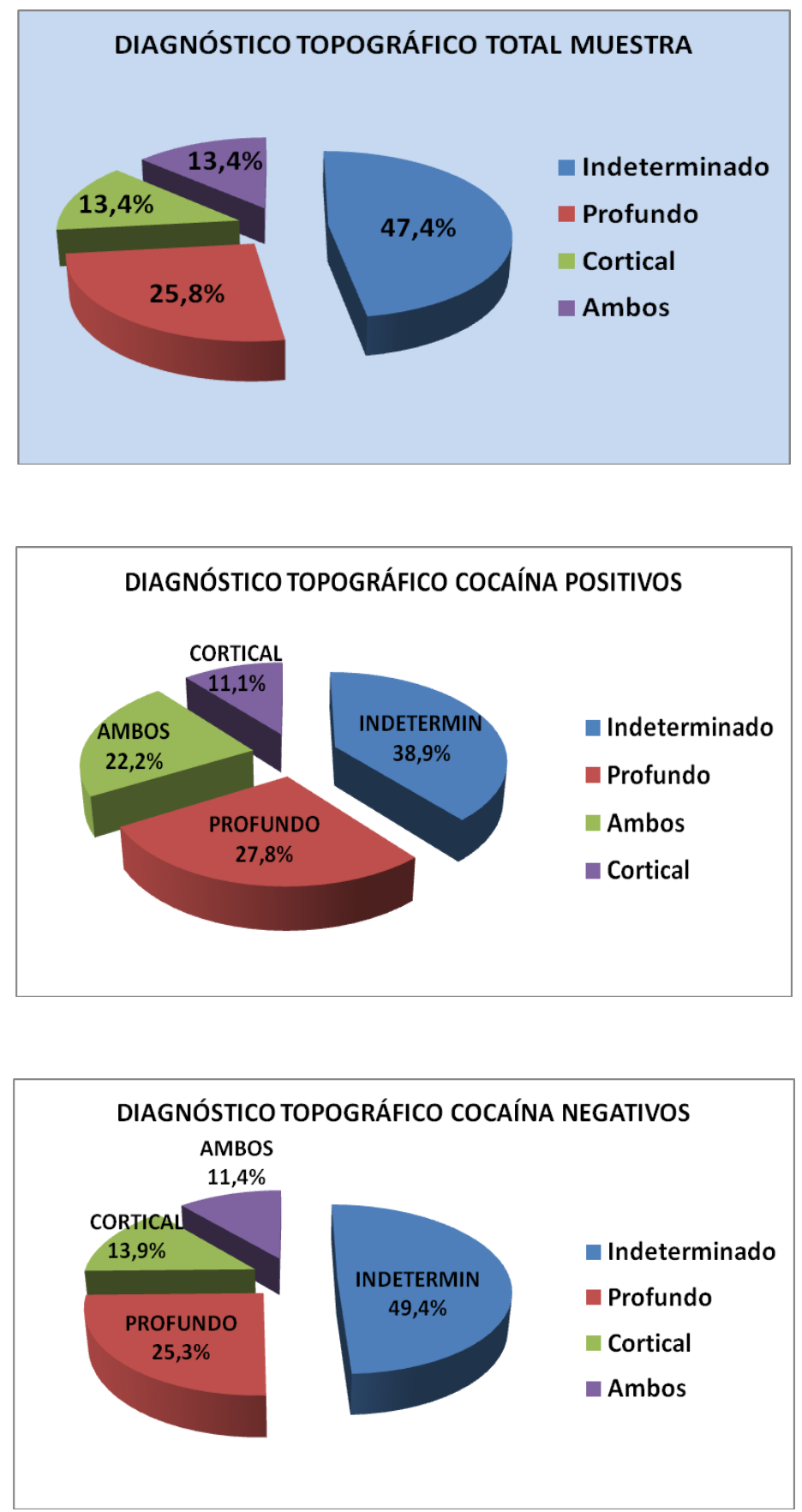

- Diagnóstico etiológico: del total de pacientes, se diagnosticó AIT en 12 (12.4\%), Ictus establecido-Hemorragia intraparenquimatosa en 15 (15.5\%), Ictus establecidoHemorragia subaracnoidea en 12 (12.4\%), Ictus establecido-Infarto cerebral aterotrombótico en 9 (9.3\%), Ictus establecido-Infarto cerebral cardioembólico en 7 (7.2\%), Ictus establecido-Infarto cerebral lacunar en 1 (1\%), Ictus establecido- 
Infarto cerebral de causa inhabitual en 20 (20.6\%), Ictus establecido-Infarto cerebral indeterminado en 21 (21.6\%), ningún caso de hemorragia cerebelosa ni intraventricular; en el grupo consumidor de cocaína, AIT en 2 (11.1\%), Ictus establecido-Hemorragia intraparenquimatosa en 4 (22.2\%), Ictus establecidoHemorragia subaracnoidea en 3 (16.7\%), Ictus establecido-Infarto cerebral de causa inhabitual en $4(22.2 \%)$, Ictus establecido-Infarto cerebral indeterminado en 5 (27.8\%), ningún caso de infarto cerebral aterotrombótico, cardioembólico ni lacunar; en el grupo control, AIT en 10 (12.7\%), Ictus establecido-Hemorragia intraparenquimatosa en 11 (13.9\%), Ictus establecido- Hemorragia subaracnoidea en 9 (11.4\%), Ictus establecido-Infarto cerebral aterotrombótico en 9 (11.4\%), Ictus establecido-Infarto cerebral cardioembólico en 7 (8.9\%), Ictus establecido-Infarto cerebral lacunar en 1 (1.3\%), Ictus establecido-Infarto cerebral de causa inhabitual en $16(20.3 \%)$, Ictus establecido-Infarto cerebral indeterminado en 16 (20.3\%).
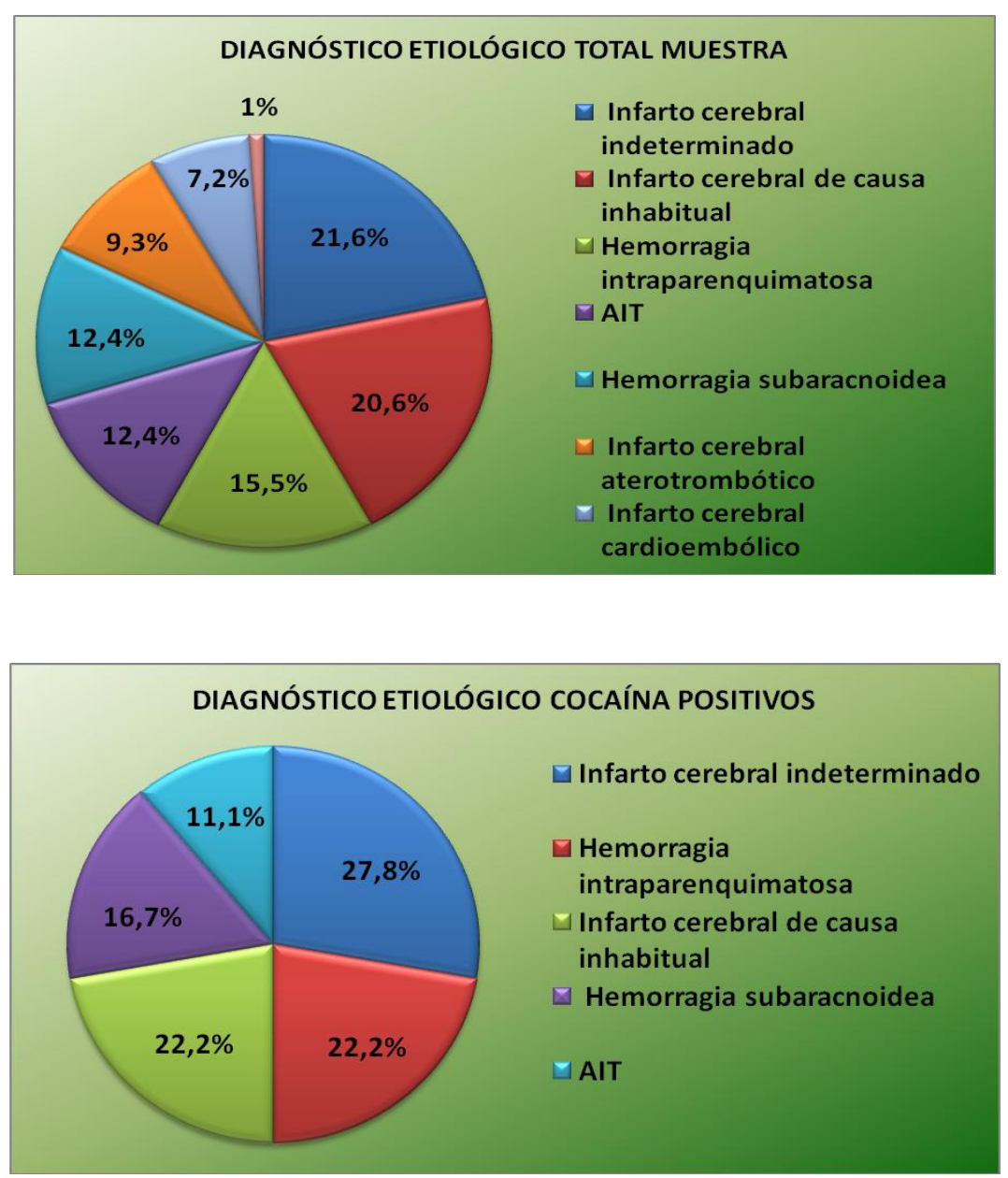


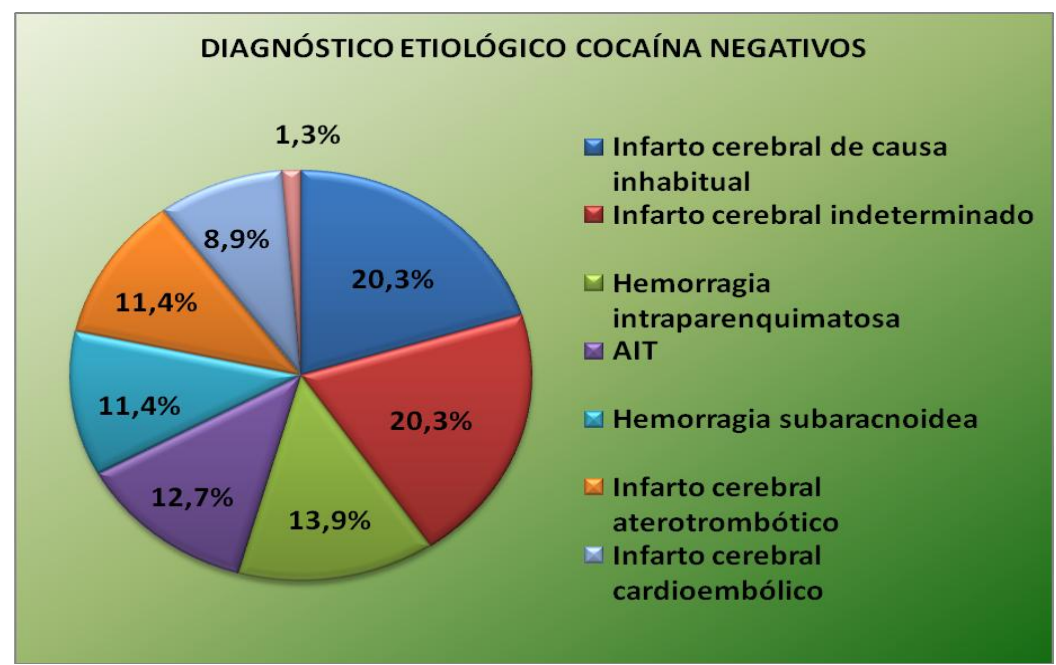

\section{Descripción de las complicaciones}

- Complicaciones: existen complicaciones neurológicas en 18 pacientes (18.6\%), 6 del grupo consumidor de cocaína (33.3\%) y 12 del grupo de control (15.2\%); además existen complicaciones sistémicas en $6(6.2 \%)$ y cardiovasculares en 3 $(3.1 \%)$, estos últimos pertenecientes al grupo control.
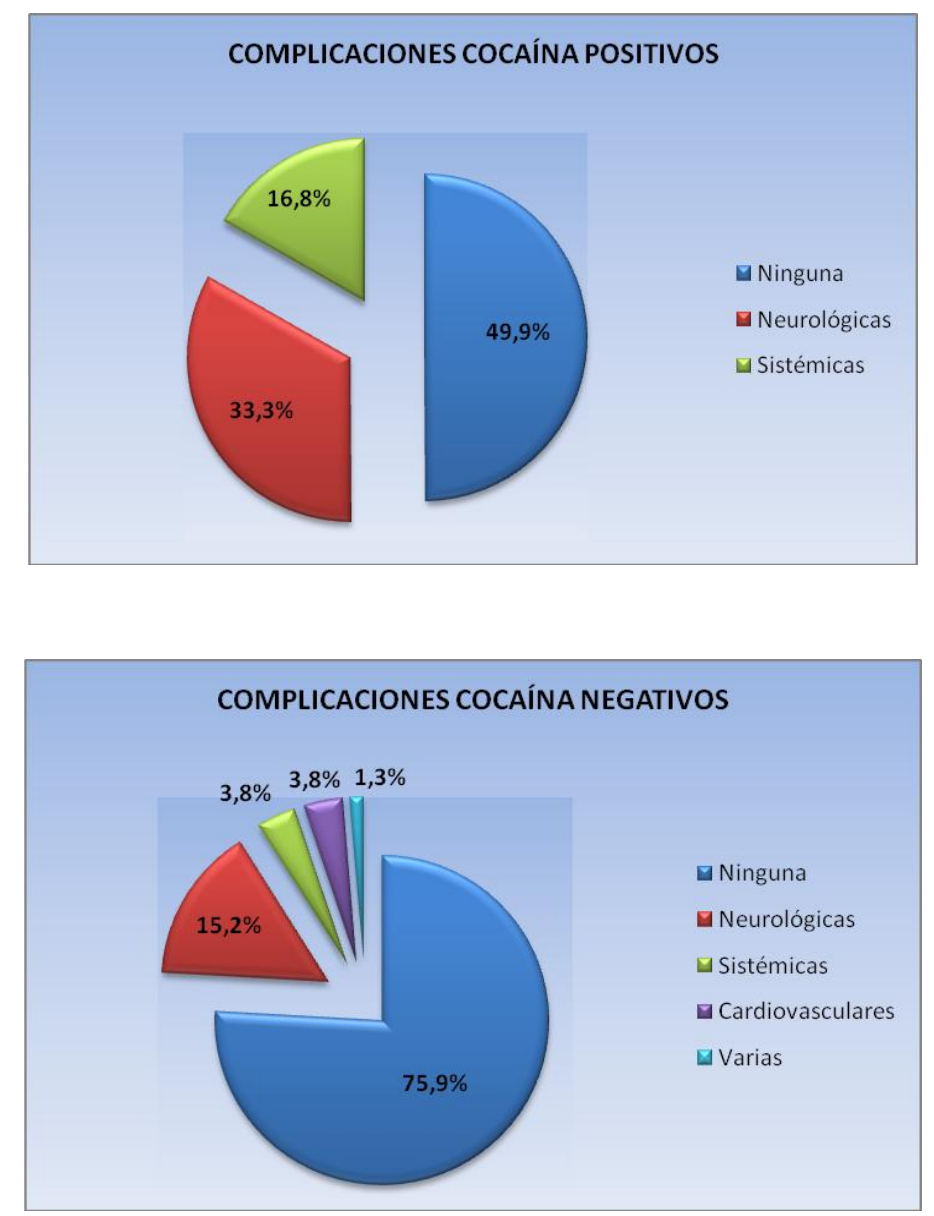
- Complicaciones neurológicas: 78 pacientes (80.4\%) no presentó ninguna complicación; las sucedidas fueron, crisis epilépticas focales en 3 (3.1\%), crisis epilépticas focal $2^{\mathrm{a}}$ generalizada en $1(1 \%)$, crisis epilépticas generalizadas en 1 (1\%), edema cerebral en 4 (4.1\%), extensión del hematoma en 1 (1\%), transformación hemorrágica no sintomática en 1 (1\%), transformación hemorrágica sintomática en $1(1 \%)$, vasoespasmo en $1(1 \%)$, progresión del ictus en $3(3.1 \%)$, recurrencia en $3(3.1 \%)$ y ningún paciente presentó status ni hidrocefalia. De todas estas complicaciones, en el grupo consumidor de cocaína, sufrieron crisis epilépticas generalizadas 1 (5.6\%), edema cerebral $2(11.1 \%)$, extensión del hematoma $1(5.6 \%)$ y crisis epilépticas focales $1(5.6 \%)$.

- Complicaciones sistémicas: 89 pacientes (91.8\%) no presentó ninguna complicación; fiebre en $2(2.1 \%)$, ITU en $2(2.1 \%)$, neumonía en $1(1 \%)$, sepsis en $2(2.1 \%)$, rabdomiolisis en 1 (1\%). De éstas complicaciones, los pacientes del grupo consumidor de cocaína, sufrieron 1 ITU (5.6\%), 1 sepsis (5.6\%) y 1 rabdomiolisis (5.6\%); en total el $16.8 \%$ frente al $6.4 \%$ del grupo control.

\section{Descripción de la morbimortalidad}

- Secuelas neurológicas: existieron secuelas en 49 pacientes del total (50.5\%), 8 del grupo consumidor de cocaína (44.4\%) y 41 del grupo control (51.8\%); no hubo secuelas neurológicas en 43 pacientes del total (44.3\%), 8 del grupo de cocaína (44.4\%) y 35 del grupo control (44.3\%) y hubo 2 éxitus (11.1\%) en el grupo de cocaína y $3(3.8 \%)$ en el grupo control.

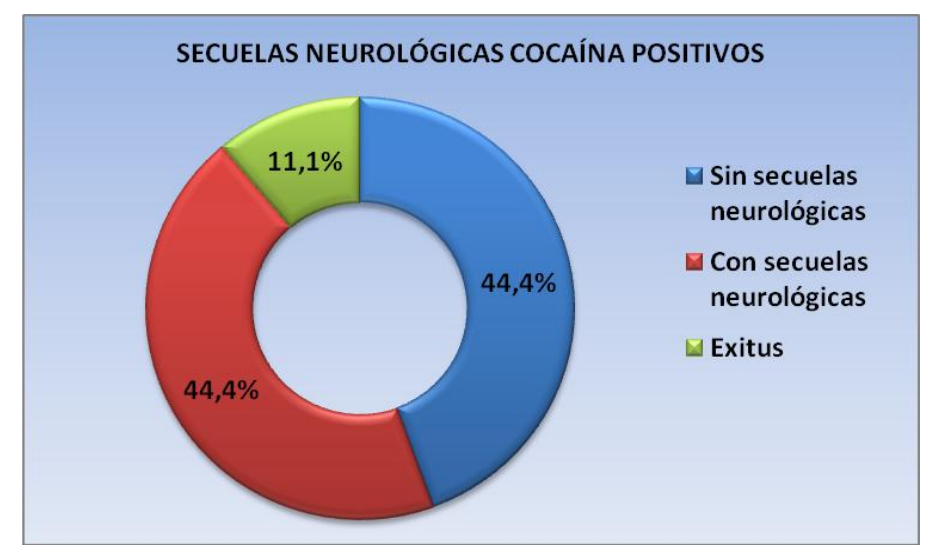




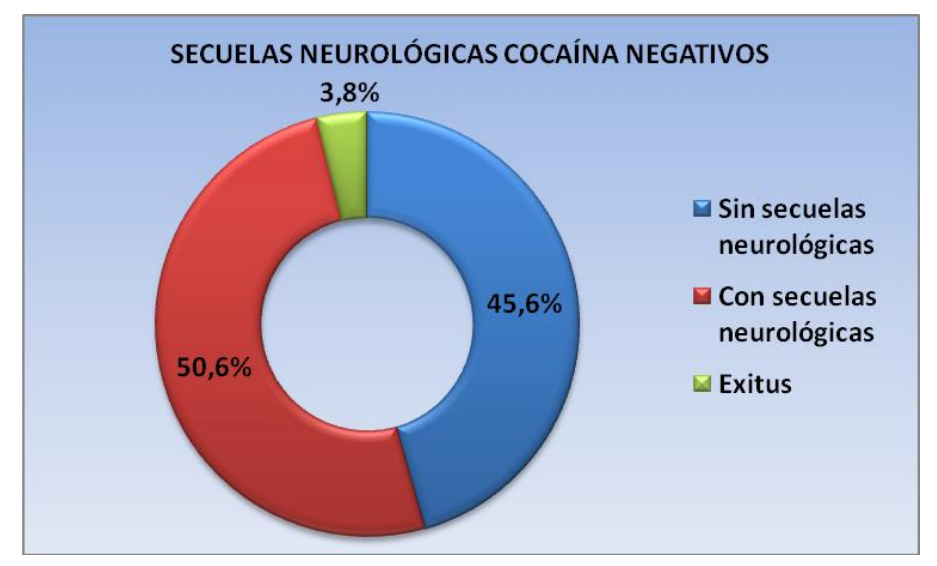

- Tipo de secuela neurológica: de los 49 pacientes que sufrieron secuelas neurológicas, 27 déficit motor (27.8\%), 5 (5.2\%) déficit sensitivo, 1 (1\%) trastorno del lenguaje y $16(16.5 \%)$ varios de los anteriores; en el grupo de cocaína hubo 8 pacientes con secuelas (44.4\%), 5 déficit motor (27.8\%), 1 (5.6\%) déficit sensitivo y $2(11.1 \%)$ varios de los anteriores; en el grupo control hubo 41 pacientes con secuelas (50.6\%), 22 déficit motor $(27.8 \%), 4(5.1 \%)$ déficit sensitivo, 1 (1.3\%) trastorno del lenguaje y $14(17.7 \%)$ varios de los anteriores.

\section{ANÁLISIS UNIVARIANTE}

Para valorar si existen diferencias estadísticamente significativas entre el grupo de pacientes consumidores de cocaína y el grupo de no consumidores, en cuanto a la edad y la estancia hospitalaria se emplea la prueba no paramétrica U de Mann-Whitney. En el análisis de la edad, como se obtiene un nivel crítico igual a 0’006 (<0’05), se rechaza la hipótesis nula de homogeneidad, por tanto, en nuestra muestra encontramos que la población de vasculares con consumo de cocaína son más jóvenes que los vasculares jóvenes sin consumo de cocaína.

Por el contrario, como al analizar la estancia hospitalaria, el nivel crítico es igual a 0’7 (>0’05), no pueden establecerse diferencias entre los grupos estudiados.

Por otro lado, se plantea si existen diferencias entre ambos grupos en cuanto a la morbimortalidad. En primer lugar, se analiza la mortalidad mediante la prueba X2 de Pearson; como el nivel crítico es $<0$ 0’01 $(<0$ ’5), rechazamos la hipótesis nula de homogeneidad, por tanto, en nuestra muestra existe diferencias significativas en cuanto a mortalidad entre ambas poblaciones, es decir, en la población de vasculares con consumo de cocaína existe mayor mortalidad que los vasculares jóvenes sin consumo de cocaína. Sin embargo al analizar la morbilidad mediante la prueba U Mann-Whitney 
sobre la variable de NIH al alta, se obtiene un nivel crítico igual a 0’784 (> 0’05), por lo que se acepta la hipótesis nula de homogeneidad, es decir, en nuestra muestra no encontramos diferencias del pronóstico funcional al alta en cuanto al déficit neurológico mediante NIHSS entre ambas poblaciones. De la misma manera, al analizar la morbilidad mediante la prueba de U de Mann-Whitney sobre la variable Rankin al alta, se obtiene un nivel crítico igual a 0’962 (>0’05), es decir, tampoco encontramos diferencias del pronóstico funcional al alta mediante escala de de Rankin entre ambas poblaciones.

Por último, dado que la HTA es uno de los factores etiopatogénicos más implicados en las complicaciones cerebrovasculares por cocaína se plantea si existen diferencias entre ambos grupos, en cuanto a antecedente de HTA ó HTA al alta; para ello se aplica de nuevo la prueba de U Mann-Whitney obteniendo resultados de 0'196 y 0’629 respectivamente ( $>0$ '05), es decir que en nuestra muestra no encontramos diferencias en cuanto a número de hipertensos entre ambas poblaciones. 


\section{DISCUSIÓN}

En nuestra muestra, existe un predominio de varones en ambos grupos, aunque la diferencia entre ambos sexos es mucho menor en el grupo consumidor de cocaína que en el grupo control; de este hecho se puede pensar que la frecuencia de patología cerebrovascular es más frecuente en varones, sobre todo, cuando no existe un factor exógeno añadido que hace disminuir esta diferencia, como en este caso el consumo de cocaína. Según las publicaciones oficiales sobre consumo de drogas [12, 13], el consumo de cocaína es más frecuente en varones, pero cada vez la diferencia entre ambos sexos se está haciendo menor.

Por otro lado, en nuestra revisión, la edad media del grupo consumidor de cocaína es menor que en el grupo control y al analizar este dato mediante la prueba no paramétrica U de Mann-Whitney, encontramos que esa diferencia es significativa. Al compararlo con otras series publicadas [11, 49], la edad media de nuestra muestra se halla en medio de los valores obtenidos en dichas series, de lo que se puede deducir que está de acorde con lo publicado.

Sobre la estancia hospitalaria no existen muchos datos publicados, y en nuestro caso, no hemos podido encontrar diferencias entre ambos grupos.

Los datos en relación al consumo de otros tóxicos también son muy dispares en la literatura [1, 2, 11, 49]. En esta revisión, podemos ver una tendencia mayor del consumo de otros tóxicos (tabaco, alcohol y cannabis) en el grupo de cocainómanos al compararlo con el grupo control; esta diferencia es evidente en el alcohol, donde el consumo es más del doble, pero sobre todo en el cannabis, ya que prácticamente el total de los pacientes que lo consumen pertenecen al grupo de cocaína. Este dato es importante, ya que indica que los pacientes tienen una tendencia al consumo de tóxicos en general, es decir, no se trata de algo ocasional o puntual, y por otra parte, destacar que sobre todo la combinación con alcohol supone un riesgo y un aumento de la morbimortalidad asociada a la cocaína, debido a la potenciación de efectos y metabolitos comunes (cocaetileno) según lo referido en la literatura $[3,8,18,19]$.

En los antecedentes médicos recogidos, cabe destacar que, en el grupo de cocaína: 1/ no existía ningún paciente con antecedente de ictus previo; 2/ la frecuencia de cefalea, a pesar de las diversas referencias que la asocian a la cocaína $[51,6,8,9]$, fue menor en este grupo; y sin embargo, 3/ la frecuencia de trastornos psiquiátricos 
(trastorno del ánimo), como era esperable, si fue mayor en éste grupo, dada la asociación de este tóxico con alteraciones neuropsicológicas y psiquiátricas [8,22].

Un apartado importante son los factores de riesgo cardiovascular (HTA, DM, DL), ya que tanto al ingreso como al alta, éstos fueron menos frecuentes en el grupo consumidor de cocaína. Al analizar cada uno de ellos por separado, llama la atención que, la mayor diferencia se encuentra en el número de pacientes hipertensos al ingreso (siendo menos de la mitad en el grupo de cocaína), pero al alta, esta diferencia disminuye en gran medida, ya que se duplica el número de hipertensos en el grupo de cocainómanos. A pesar de que al analizar esta variable con la prueba de U MannWhitney, no existen diferencias significativas; una posible explicación a esta diferencia, sería que la HTA detectada en los pacientes consumidores de cocaína estuviera en relación al mecanismo adrenérgico y por tanto elevador de la presión arterial que se asocia a la cocaína $[3,44,17]$; además no podemos obviar que durante la fase aguda del ictus existe una tendencia a la HTA. Sería interesante comprobar la evolución posterior de estos pacientes y si las cifras tensionales se normalizan si cesa el consumo del tóxico.

En los pacientes consumidores de cocaína, también parece existir una tendencia a presentar cifras elevadas de creatín-kinasa, mayor que en el grupo control; este fenómeno podría ser otra consecuencia de la estimulación simpática y compatible con diversos artículos publicados que establecen esta asociación.

En cuanto al resto de pruebas complementarias dirigidas al estudio etiológico del ictus, puede verse una tendencia hacia mayor normalidad de las mismas, en el grupo de consumidores de cocaína. Es decir, menos hallazgos patológicos en el estudio vascular, ecocardiograma y estudio de trombosis. Además menor frecuencia de shunt derecha izquierda y ningún caso de fibrilación auricular ni disección arterial. Aunque debido al tamaño de la muestra y limitaciones del estudio, no podemos establecer esta relación como cierta, si podemos decir que, en nuestra muestra existe esta tendencia; a partir de aquí podemos plantear, que en los casos donde el estudio etiológico es normal, la cocaína puede ser la responsable o al menos un factor importante en la aparición del evento cerebrovascular agudo.

En ambos grupos de nuestra muestra, son más frecuentes los eventos isquémicos que hemorrágicos, si bien es cierto, que en el grupo consumidor de cocaína son más frecuentes los hemorrágicos en relación al grupo control. Como se comentó previamente, este dato no está aclarado todavía, y varía de unas series a otras [11, 25, 49]. Como diagnóstico clínico, el más frecuente en ambos grupos es el síndrome lacunar. No existe 
muchos datos en la literatura en cuanto al diagnóstico topográfico, pero en nuestra serie, aunque existe una tendencia a mayor afectación del territorio anterior en ambos grupos, ésta es mayor en el grupo de cocaína y sobre todo es evidente que existe menor afectación del territorio posterior; este fenómeno, al que se hace referencia en algunos artículos $[11,16]$, puede estar en relación a la inervación simpática y distribución de los receptores postsinápticos en cada territorio arterial.

Por otro lado, no existen diferencias destacables entre ambos grupos en la localización profunda o cortical de las lesiones. El diagnóstico etiológico de nuestra muestra se establece mediante criterios de la clasificación TOAST; en los resultados existe un importante número de pacientes con infarto cerebral indeterminado por 2 o más causas o por estudio incompleto, que no se recoge en otras series. La frecuencia de hemorragia intraparenquimatosa es más frecuente que la hemorragia subaracnoidea en ambos grupos, similar a resultados de otras series [11, 49] y a su vez, ambas son más frecuentes en el grupo consumidor de cocaína. Sobre HSA asociadas a cocaína y el pronóstico de éstas se han publicado varios artículos [23, 24, 26, 39]; en nuestra muestra la proporción de aneurismas es mayor en el grupo de cocaína y ninguna de ellas tiene complicaciones ni secuelas excepto un caso del grupo de cocaína que sufre vasoespasmo y queda con secuelas. La frecuencia de AIT es similar en ambos grupos y merece la pena destacar que en el grupo de cocaína no existe ningún ictus que mediante esta clasificación se pueda catalogar como cardioembólico ni aterotrombótico.

Por último, en el grupo consumidor de cocaína existe mayor número de complicaciones neurológicas y el análisis estadístico muestra diferencias significativas en cuanto a mortalidad que no se encuentran en cuanto a morbilidad tanto mediante escala de NIH como Rankin al alta. Esto puede hacernos pensar que la mayoría de ictus en pacientes jóvenes tienen un pronóstico similar en lo que se refiere a morbilidad, independientemente de que haya consumo o no de cocaína, pero que este tóxico, por sus diversos mecanismos de acción puede provocar ictus y hemorragias graves que provoquen la muerte precoz del paciente [49]. 


\section{CONCLUSIONES}

1. La cocaína es un factor de riesgo evidente a tener en cuenta en adultos jóvenes, aún en presencia de otros factores de riesgo vascular.

2. La cocaína presenta como órganos diana el sistema cardiovascular y el sistema nervioso predominantemente.

3. Según los datos epidemiológicos, existe un aumento del consumo de cocaína y a edades más tempranas.

4. El consumo de este tóxico, parece responsable de eventos neurológicos a edades más tempranas de lo esperado según la evolución natural con los habituales factores de riesgo vascular, lo cual está provocando una morbilidad secundaria importante.

5. Existen diversos mecanismos implicados entre los que destacan el vasoespasmo, aumento de presión arterial y alteración de neurotransmisores.

6. Los ictus asociados a cocaína se asocian a mayor mortalidad precoz, pero no hemos podido concluir que lo hagan a mayor morbilidad.

7. Existe un predominio de lesiones en circulación anterior y de tipo isquémico.

8. Sería recomendable realizar estudio toxicológico en orina a todos los pacientes jóvenes con sintomatología neurológica, en especial ictus, ya que es una prueba inocua y sencilla, que puede aportar una información de gran valor diagnóstico, pronóstico y terapéutico.

9. La concienciación de los diferentes especialistas, en este caso neurólogos, de los efectos de la cocaína es fundamental para poder diagnosticar las complicaciones secundarias a su consumo.

10. Consideramos importante realizar estudios prospectivos sobre este tema, por la implicación sociosanitaria que tiene, y la controversia existente en algunos puntos concretos de los resultados del escaso número de trabajos publicados. 


\section{BIBLIOGRAFÍA}

1. Pascual Pastor, F. Aproximación histórica a la cocaína. De la coca a la cocaína. Monografía de cocaína. Adicciones 2001; 3, (supl 2): 7-22.

2. Bobes, J. Sáiz, P.A, González, M.P, Bascarán, M.T. Epidemiología del uso/abuso de cocaína. Monografía de cocaína. Adicciones 2001; 3, (supl 2): 23-36.

3. Lizasoain, I.; Moro, M.A.; Lorenzo, P. Cocaína: aspectos farmacológicos. Monografía de cocaína. Adicciones 2001; 3, (supl 2): 37-45.

4. E. Fernández-Espejo. Neurobiología de la adicción a psicoestimulantes. Rev Neurol 2006; 43: 147-54.

5. Llopis Llácer, J.J. Dependencia, intoxicación aguda y síndrome de abstinencia por cocaína. Monografía de cocaína. Adicciones 2001; 3, (supl 2): 147-165.

6. Balcells Oliveró, M. Complicaciones orgánicas de la cocaína. Monografía de cocaína. Adicciones 2001; 3, (supl 2): 167-177.

7. Solé Puig, J. Tratamiento del consumo de cocaína. Integrando psicoterapia y farmacoterapia. Monografía de cocaína. Adicciones 2001; 3, (supl 2): 209-225.

8. Torres Hernández MA, Climent Díaz B. Complicaciones neurológicas por cocaína. Monografía patología orgánica en adicciones. Adicciones 2006; 18, (supl 1): 179196.

9. Neiman J, Haapaniemi HM, Hillbom M. Neurological complications of drug abuse: pathophysiological mechanisms. Eur J Neurol 2000; 7:595-606.

10. Martin-Schild S, Albright KC, Misra V, Philip M, Barreto AD, Hallevi H, Grotta JC, Savitz SI. Intravenous tissue plasminogen activator in patients with cocaineassociated acute ischemic stroke. Stroke. 2009; 40:3635-7.

11. Blanco M, Diez-Tejedor E, Vivancos F, Barreiro P. Cocaine and cerebrovascular disease in Young adults. Rev Neurol 1999;29:796-800.

12. Observatorio europeo de las drogas y las toxicomanías. El problema de la drogodependencia en Europa. Luxemburgo: Oficina de Publicaciones de la Unión Europea. Informe anual 2009; 67-79.

13. Obsevatorio español de drogas 2007. Encuesta domiciliaria sobre abuso de drogas en España (EDADES). Ministerio de Sanidad y Consumo.

14. Ritz MC, Lamb RJ, Goldberg SR, Kuhar MJ. Cocaine receptors on dopamine transporters are related to self-administration of cocaine. Science 1987; 237: 121923.

15. Kalivas PW, Duffy P. Repeated cocaine administration alters extracellular glutamate in the ventral tegmental area. J Neurochem 1998; 70:1497-502.

16. DíezTejedor E, Tejada J, Muñoz J. Cerebral arterial changes following cocaine iv administration: an angiographic study in rabbits. J Neurol 1992; 239 (Suppl 2): S38.

17. Konzen JP, Levine SR, García JH. Vasospasm and thrombus formation as a possible mechanism of stroke related to alkaloidal cocaine. Stroke 1995; 26: 11148.

18. Bolouri MR, Small GA. Cerebrovascular and cardiovascular complications of alcohol and sympathomimetic drug abuse. Med Clin North Am. 2005; 89: 1343 1358.

19. Gottschalk PC, Kosten TR. Cerebral perfusion defects in combined cocaine and alcohol dependence. Drug Alcohol Depend. 2002; 69: 95-104

20. Rowley HA, Lowenstein DH, Rowbotham MC, Simon RP. Thalamomesencephalic strokes after cocaine abuse. Neurology 1989; 39: 42830. 
21. Kaufman MI, Levin JM, Ross MH, Lange N, Rose SL, Kukes TJ, et al. Cocaine induced cerebral vasoconstriction detected in humans with MR angiography. JAMA 1998; 279: 376-380.

22. A. Verdejo-García a, b, M. Pérez-García a, b, M. Sánchez-Barrera a, A. RodríguezFernández c, M. Gómez-Río c. Neuroimagen y drogodependencias: correlatos neuroanatómicos del consumo de cocaína, opiáceos, cannabis y éxtasis. Rev Neurol 2007; 44: 432-439.

23. Vannemreddy P, Caldito G, Willis B, Nanda A. Influence of cocaine on ruptured intracranial aneurysms: a case control study of poor prognostic indicators.

24. J Neurosurg. 2008; 108:470-6.

25. Nanda A, Vannemreddy PS, Polin RS, Willis BK. Intracranial aneurysms and cocaine abuse: analysis of prognostic indicators. Neurosurgery. 2000; 46:1063-7.

26. Toossi S, Hess CP, Hills NK, Josephson SA. Neurovascular Complications of Cocaine Use at a Tertiary Stroke Center. Department of Neurology, University of California San Francisco. J Stroke Cerebrovasc Dis. 2010 May 3. [Epub ahead of print].

27. Conway JE, Tamargo RF. Cocaine use is an independent risk factor for cerebral vasospasm after aneurismal subarachnoid haemorrhage. Stroke. 2001; 32: 2338.

28. Pentkowski NS, Acosta JI, Browning JR, Hamilton EC, Neisewander JL. Stimulation of 5-HT (1B) receptors enhances cocaine reinforcement yet reduces cocaine-seeking behavior. Addict Biol. 2009; 14:419-30.

29. Lucas CE. The impact of street drug son trauma care. J Trauma. 2005; 59: 57-60.

30. Wu YW et al. Perinatal arterial stroke: understanding mechanisms and outcomes. Semin Neurol. 2005; 25: 424-434.

31. Martin-Schild S, Albright KC, Hallevi H, Barreto AD, Philip M, Misra V, Grotta JC, Savitz SI. Intracerebral hemorrhage in cocaine users. Stroke. 2010; 41:680-4.

32. DíezTejedor E, Frank A, Gutiérrez M, Barreiro P. Encephalopathy and biopsyproven cerebrovascular inflammatory changes in a cocaine abuser. Eur $J$ Neurol 1998; 5: 1037

33. Johnson BA, et al. Treatment advances for cocaine induced ischemic stroke: focus on dyhidropiridine-class calcium channel antagonists. Am J Psychiatry. 2001; 158: 1191-1198.

34. Johnson BA, et al. Isradipine decreases the hemodynamic response of cocaine and methamphetamine, results from two human laboratory studies. Am J Hypertens. 2005; 18: 813-822.

35. Johnson BA, et al. Acute intravenous lowand- high-dose cocaine reduces quantitative global and regional cerebral blood flow in recently abstinent subjects with cocaine use disorder. J Cereb Blood Flow Metab. 2005; 25: 928-936

36. Kosten TR. Pharmacotherapy of cerebral ischemia in cocaine dependence. Drug Alcohol Depend. 1998; 49: 133-144.

37. Su J, et al. Cocaine induces apoptosis in cerebral vascular muscle cells: potential roles in strokes and brain damage. Eur J Pharmacol. 2003; 482: 61-66.

38. Vallee JN, et al. Acute basilar artery occlusion treated by bhromboaspiration in a cocaine and ecstasy abuser. Neurology. 2003; 61: 839-841.

39. Aggarwal SK, Williams V, Levine SR, Cassin BJ, Garcia JH. Cocaine-associated intracranial hemorrhage: absence of vasculitis in 14 cases. Neurology. 1996; 46:1741-3.

40. Broderick JP, et al. Major risk factors for aneurysmal subarachnoid hemorrhage in the young modifiable. Stroke. 2003; 34: 1375-1381. 
41. Feldmann E, Broderick JP, et al. Major risk factors for intracerebral haemorrhage in the young are modifiable. Stoke. 2005; 36: 1881-1885.

42. Licata SC, Renshaw PF. Neurochemistry of drug action: insights from proton magnetic resonance spectroscopic imaging and their relevance to addiction. Ann NY Acad Sci. 2010; 1187:148-71.

43. Kosten TR, Tucker K, Gottschalk PC, Rinder CS, Rinder HM. Platelet abnormalities associated with cerebral perfusion defects in cocaine dependence. Biol Psychiatry. 2004; 55:91-7.

44. Fredericks RK, Lefkowitz DS, Challa VR. Cerebral vasculitis associated with cocaine abuse. Stroke. 1991; 22:1437-1439

45. Jacobs I, Myer H, Rosler M, et al. Cocaine abuse: neurovascular complications. Radiology 1989; 170: 2237.

46. DíezTejedor E, Tejada J, Frank A. Complicaciones neurológicas debidas al consumo de cocaína, anfetaminas y simpaticomiméticos. Arch Neurobiol 1989; 52 (Suppl 1): 16282.

47. Bartzokis G, Beckson M, Hance DB, Lu PH, Foster JA, Mintz J, Ling W, Bridge P. Magnetic resonance imaging evidence of "silent" cerebrovascular toxicity in cocaine dependence. Biol Psychiatry. 1999; 45: 1203-1211.

48. Heesch CM, Wilhelm CR, Ristich J, Adnane J, Bontempo FA, Wagner WR. Cocaine activates platelets and increases the formation of circulating platelet containing microaggregates in humans. Heart. 2000; 83: 688-695.

49. Williams JB, et al. Hemodynamic response profile predicts susceptibility to cocaineinduce toxicity. Eur J Pharmacol. 2003; 464: 189-196.

50. Nanda A, Vannemreddy P, Willis B, Kelley R. Stroke in the young: relationship of active cocaine use with stroke mechanism and outcome. Acta Neurochir Suppl. 2006; 96:91-6.

51. Penarrocha M, Bagan JV, Penarrocha MA, Silvestre FJ. Cluster headache and cocaine use. Oral Surg Oral Med Oral Pathol Oral Radiol endod 2000; 90: 271 274.

52. Dhuna A, Pascual_Leone A, Belgrade M. Cocaine-related vascular headaches. J Neurol Neurosurg Psychiatry. 1991; 54: 807- 812. 


\section{ANEXO}

\section{ESCALAS}

\section{Clasificación OCSP}

\section{(Oxfordshire Stroke Project Classication)}

Basada en la localización clínica y tipo isquemia/hemorragia, correlación con el pronóstico e índice de recurrencias (Bamford J; Lancet 1991, Mead G, JNNP 2000)

Nota: En la presente revisión sólo hemos utilizado esta clasificación para los ictus isquémicos; en hemorragias después del TC no se utilizan estos criterios clínicos.

\section{Denominación General}

- TAC: Total Anterior Circulation (Stroke)

- PAC: Partial Anterior Circulation (Stroke)

- LAC: Lacunar (Stroke)

- POC: Posterior Circulation (Stroke)

Clasificación final: se expresa en cuatro letras.

\section{Definiciones:}

- TACI: Infarto total de la circulación anterior, cumpliendo los tres siguientes criterios:

○ Disfunción cerebral cortical, afasia, discalculia, alteraciones visuoespaciales

- Déficit motor y/o sensitivo en al menos dos de tres áreas corporales (CARA, MMSS, MMII).

○ Hemianopsia homónima

- PACI: Infarto parcial de la circulación anterior, cuando cumple alguno de los siguientes criterios:

○ Disfunción cerebral cortical afasia, discalculia, alteraciones visuoespaciales

- Dos de los tres criterios de TACI

○ Déficit motor y/o sensitivo más restringido que el clasificado como LACI ( limitado a una extremidad )

- LACI: infarto lacunar: cuando n existe déficit cortical ni hemianopsia, y se cumple uno de los siguientes criterios: 
○ Síndrome motor puro que afecta a dos de tres áreas corporales (cara, MMSS, MMII).

○ Síndrome sensitivo puro que afecta a dos de tres áreas

- Síndrome sensitivo motor puro que afecta a dos de tres áreas.

○ Hemiparesia-ataxia ipsilateral

○ Disartria-mano torpe

○ Movimientos anormales focales y agudos (hemicorea, hemibalismo).

- POCI: Infarto en la circulación posterior, cuando se cumple alguno de los criterios:

- Afectación ipsilateral de pares craneales con déficit motor y/o sensitivo ipsilateral

○ Déficit motor y/o sensitivo bilateral

○ Enfermedad oculomotora

- Disfunción cerebelosa sin déficit de vías largas ipsilaterales ( lo que sería un síndrome lacunar de hemiparesia-ataxia)

○ Hemianopsia homónima aislada.

\section{Escala RANKIN}

$0 \rightarrow$ Sin síntomas.

$1 \rightarrow$ Sin incapacidad importante: Capaz de realizar sus actividades y obligaciones habituales.

$2 \rightarrow$ Incapacidad leve: Incapaz de realizar algunas de sus actividades previas, pero capaz de velar por sus intereses y asuntos sin ayuda.

$3 \rightarrow$ Incapacidad moderada: Síntomas que restringen significativamente su estilo de vida o impiden su subsistencia totalmente autónoma (p. ej. necesitando alguna ayuda).

$4 \rightarrow$ Incapacidad moderadamente severa: Síntomas que impiden claramente su subsistencia independiente aunque sin necesidad de atención continua (p. ej. incapaz para atender sus necesidades personales sin asistencia).

$5 \rightarrow$ Incapacidad severa: Totalmente dependiente, necesita asistencia constante día y noche.

$6 \rightarrow$ Muerte 
Escala NIHSS (National Institute of Health Stroke Scale)

Adaptación al Español. J. Montaner y J Alvarez-Sabin. Neurología, 2006 21(4). 192202.

Escala de Ictus del National Institute of Health

\begin{tabular}{|c|c|c|c|}
\hline NIVEL DE CONCTENCTA & & PIERNA IZQUIERDA & \\
\hline - Alerta & $\mathbf{0}$ & - No claudica BM 5 & $\mathbf{0}$ \\
\hline Somndiento & 1 & - Claudica (BM 4) & 1 \\
\hline - Estuparaso & 2 & - Algún esfuerza contra gravedad (BM 3) & 2 \\
\hline - Coma & 3 & - Sin esfuerza contra grạm & 3 \\
\hline PREGUNTAS LOC & & - Ningún mavimienta (BM O) & 4 \\
\hline - Responde ambas comrectamente & $\mathbf{o}$ & PIERNA DERECHA & \\
\hline - Responde una correctamente & 1 & - No claudica BM 5 & $\mathbf{0}$ \\
\hline Incorrecto & 2 & - Claudica (BM 4) & 1 \\
\hline ORDENES LOC & & - Algún esfuerza contra gravedad (BM 3) & 2 \\
\hline - Realiza ambas correctamente & & - Sin esfuerza contra gravedad (BM 2-1) & 3 \\
\hline - Realiza una correctamente & & - Ningún mavimienta (BM O) & 4 \\
\hline - Incorrecto & & ATAXIA DE MIEMBROS & \\
\hline MIRADA & & Ausente & o \\
\hline Narmal & $\mathbf{o}$ & - Presente en una extremidad & 1 \\
\hline - Parálisis parcial de la mirada & 1 & - Presente en dos extremidades & 2 \\
\hline - Desviación Aculacefálica & 2 & 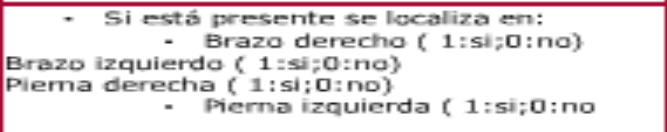 & \\
\hline CAMPOS VISUALES & & SENSTBILIDAD & \\
\hline - Sin defectos campimétricos & $\mathbf{a}$ & - Normal & $\mathbf{0}$ \\
\hline - Cuadranapsia & 1 & - Hipoestesia ligera a moderada & 1 \\
\hline Hernianapsia homónima & 2 & Hipdestesia severa o anestesia & 2 \\
\hline - Hemianapsia hamb́nima bilateral & 3 & LENGUAJE & \\
\hline
\end{tabular}

\begin{tabular}{|c|c|c|c|}
\hline (ceguera cortical) & & & \\
\hline PARALISIS FACIAL & & Nommal, sin afasia & 0 \\
\hline - Movimientas normales y simétricas & $\mathbf{o}$ & - Afasia ligera a moderada & 1 \\
\hline - Paresia ligera & 1 & - Arasia severa, Broca, Wernicke & 2 \\
\hline - Paresia parcial & 2 & - Afasia Global a mutismo & 3 \\
\hline - Paresia severa o parálisis total & 3 & DISARTRIA & \\
\hline BRAZO IZQUTERDO & & - Articulación Normal & 0 \\
\hline - Na claudica BM 5 & o & - Disartria leve a maderada & 1 \\
\hline - Claudica (BM 4) & $\mathbf{1}$ & - Disartria severa, anartria & 2 \\
\hline - Algún esfuerza contra gravedad (BM 3) & 2 & EXTINCTON & \\
\hline - Sin esfuerza contra gravedad (BM 2-1) & 3 & Normal & $\mathbf{0}$ \\
\hline - Ningún mavimiento (BM O) & 4 & - Parcial f sóla una modalidad afectada & 1 \\
\hline BRAZO DERECHO & & - Completa ( más de una modalidad) & 2 \\
\hline Na claudica BM 5 & o & & \\
\hline - Claudica (BM 4) & 1 & & \\
\hline - Algún esfuerzo contra gravedad (BM 3) & 2 & & \\
\hline - Sin esfuerza contra gravedad (BM 2-1) & 3 & & \\
\hline - Ningún mavimiento (BM O) & 4 & & \\
\hline
\end{tabular}




\section{Tipos Etiológicos y criterios diagnósticos (TOAST)}

\section{Clasificación de los infartos cerebrales en sus diferentes subtipos etiológicos}

1. Infarto aterotrombótico. Aterosclerosis de arteria grande. Infarto generalmente de tamaño medio o grande, de topografía cortical o subcortical y localización carotídea o vertebrobasilar, en el que se cumple alguno de los dos criterios siguientes:

A. Aterosclerosis con estenosis: estenosis mayor o igual al 50\% del diámetro luminal u oclusión de la arteria extracraneal correspondiente o de la arteria intracraneal de gran calibre (cerebral media, cerebral posterior o tronco basilar), en ausencia de otra etiología.

B. Aterosclerosis sin estenosis: presencia de placas o de estenosis inferior al 50\% en la arteria cerebral media, cerebral posterior o basilar, en ausencia de otra etiología y en presencia de al menos dos de los siguientes factores de riesgo vascular cerebral: edad mayor de 50 años, hipertensión arterial, diabetes mellitus, tabaquismo o hipercolesterolemia.

2. Infarto Cardioembólico. Infarto generalmente de tamaño medio o grande, de topografía habitualmente cortical, en el que se evidencia, en ausencia de otra etiología, alguna de las siguientes cardiopatías embolígenas: presencia de un trombo o un tumor intracardiaco, estenosis mitral reumática, prótesis aórtica o mitral, endocarditis, fibrilación auricular, enfermedad del nodo sinusal, aneurisma ventricular izquierdo o acinesia después de un infarto agudo de miocardio, infarto agudo de miocardio(menos de 3 meses), o presencia de hipocinesia cardíaca global o discinesia.

3. Enfermedad oclusiva de pequeño vaso arterial. Infarto lacunar. Infarto de pequeño tamaño(menor de $1.5 \mathrm{~cm}$ de diámetro) en el territorio de una arteria perforante cerebral, que habitualmente ocasiona clínicamente un síndrome lacunar (hemiparesia motora pura, síndrome sensitivo puro, síndrome sensitivo-motriz, hemiparesia-ataxia y disartria-mano torpe)en un paciente con antecedente personal de hipertensión arterial u otros factores de riesgo cerebrovascular, en ausencia de otra etiología.

4. Infarto cerebral de causa inhabitual. Infarto de tamaño pequeño, mediano o grande, de localización cortical o subcortical, en territorio carotídeo o vertebrobasilar en 
un paciente en el que se ha descartado el origen aterotrombótico, cardioembólico o lacunar. Se suele producir por enfermedades sistémicas (conectivopatía, infección, neoplasia, síndrome mieloproliferativo, alteraciones metabólicas, de la coagulación...) o por otras enfermedades como: disección arterial, displasia fibromuscular, aneurisma sacular, malformación arteriovenosa, trombosis venosa cerebral, angeítis, migraña, etc.

5. Infarto cerebral de origen indeterminado. Infarto de tamaño medio o grande, de localización cortical o subcortical, en territorio carotídeo o vertebrobasilar, en el que tras un exhaustivo estudio diagnóstico, han sido descartados los subtipos aterotrombótico, cardioembólico, lacunar y de causa inhabitual, o bien coexistía más de una posible etiología. Dentro de esta etiología indeterminada se podría plantear unas subdivisiones que aclararían mejor este apartado; estudio incompleto, más de una etiología y desconocida. 
